\title{
APPLICATION OF A GLOBAL IMPLICIT FUNCTION THEOREM TO A GENERAL FRACTIONAL INTEGRO-DIFFERENTIAL SYSTEM OF VOLTERRA TYPE
}

\author{
DARIUSZ IDCZAK AND STANISLAW WALCZAK
}

Communicated by Juan J. Trujillo

\begin{abstract}
In this paper, we use a global implicit function theorem for the investigation of the existence and uniqueness of a solution as well as the sensitivity of a Cauchy problem for a general integro-differential system of order $\alpha \in(0,1)$ of Volterra type, involving two functional parameters nonlinearly.
\end{abstract}

1. Introduction. Integro-differential systems have recently been studied by Aghajani et al. [1], Ahmad and Nieto [2], Bushnaq et al. [6], Gayathri et al. [8], Matar [19], Nazari and Shahmorad [20], Sudsutad and Tariboon [22], Wang and Wei [23] and Yan [26]. These systems are investigated in finite and infinite dimensional spaces, with RiemannLiouville and Caputo derivatives as well as with different types of initial and boundary conditions: local, nonlocal, involving values of solutions or their fractional integrals, delay. Tools used in studies of such systems also are of different types: Banach, Brouwer, Schauder, Schaefer, Krasnoselskii fixed point theorems, nonlinear alternative Leray-Schauder type, fractional differential transform method, strongly continuous operator semigroups, and the reproducing kernel Hilbert space method.

In [15], a global inverse function theorem obtained in [14] has been applied to the Cauchy problem:

2010 AMS Mathematics subject classification. Primary 26A33, 34K37, 47J07.

Keywords and phrases. Fractional derivatives, integro-differential equations, sensitivity, global implicit function theorem.

The project was financed with funds from the National Science Centre, granted on the basis of decision DEC-2011/01/B/ST7/03426.

Received by the editors on March 5, 2015, and in revised form on July 15, 2015. DOI:10.1216/JIE-2015-27-4-521

Copyright (C)2015 Rocky Mountain Mathematics Consortium 


$$
\left\{\begin{array}{l}
D_{a+}^{\alpha} x(t)+\int_{0}^{t} \Phi(t, \tau, x(\tau)) d \tau=v(t), \quad t \in J:=[a, b] \text { a.e. } \\
I_{a+}^{1-\alpha} x(a)=0
\end{array}\right.
$$

where $v \in L_{n}^{2}=L^{2}\left(J, \mathbb{R}^{n}\right)$ is a functional parameter and $x \in I_{a+}^{\alpha}\left(L_{n}^{2}\right)$ is an unknown function. More precisely, sufficient conditions for the existence, uniqueness and continuous differentiability of the mapping

$$
L_{n}^{2} \ni v \longmapsto x_{v} \in I_{a+}^{\alpha}\left(L_{n}^{2}\right)
$$

where $x_{v}$ is a unique solution to problem (1.1), corresponding to $v$, have been formulated. The continuous differentiability of the above mapping is often called sensitivity of the problem.

The aim of the present paper is to derive results of such a type for a more general problem, namely,

$$
\left\{\begin{array}{l}
D_{a+x}^{\alpha} x(t)+\int_{a}^{t} \Phi(t, \tau, x(\tau), u(\tau)) d \tau=f(t, x(t), v(t)), \quad t \in J \text { a.e. } \\
I_{a+}^{1-\alpha} x(a)=0
\end{array}\right.
$$

where $u \in L_{m}^{\infty}=L^{\infty}\left(J, \mathbb{R}^{m}\right)$ and $v \in L_{r}^{\infty}=L^{\infty}\left(J, \mathbb{R}^{r}\right)$ are functional parameters, involved nonlinearly, and $x \in I_{a+}^{\alpha}\left(L_{n}^{2}\right)$ is an unknown function. Equation (1.2) can be the basic system for an integrodifferential fractional games theory.

It is worth noting (cf., [3]) that, if $\alpha \in(1 / 2,1)$, then any function $x \in I_{a+}^{\alpha}\left(L_{n}^{2}\right)$ satisfies the condition $x(a)=0$. Consequently, in such a case, each solution of problem (1.2) satisfies the additional condition $x(a)=0$.

To study the existence, uniqueness and continuous differentiability of the mapping

$$
L_{m}^{\infty} \times L_{r}^{\infty} \ni(u, v) \longmapsto x_{u, v} \in I_{a+}^{\alpha}\left(L_{n}^{2}\right),
$$

where $x_{u, v}$ is a unique solution to (1.2), corresponding to the pair of functional parameters $(u, v)$, we apply a new method, based on a global implicit function theorem derived in [10]. Such a method has been applied in [10] to an integro-differential equation of the first order, involving parameter $u$ nonlinearly and parameter $v$ linearly, and to the classical differential equation of the first order, containing one parameter $u$ nonlinearly. 
In [11], we obtained some strengthening of the global implicit function theorem derived in [10]. Shortly speaking, we replaced the "bijectivity" condition by a "nonorthogonality" one. An open problem is to formulate assumptions on $\Phi$ and $f$ such that the strengthened version of the global implicit function theorem is applicable to problem (1.2) whereas the theorem from [10] is not.

An extension of the global inverse function theorem, obtained in [14], to the case of the Banach range space, has been obtained in [7] and applied to the problem

$$
\left\{\begin{array}{l}
x^{\prime}(t)+\int_{a}^{t} \Phi(t, \tau, x(\tau)) d \tau=v(t), \quad t \in J \text { a.e. } \\
x(a)=0 .
\end{array}\right.
$$

To our best knowledge, global sensitivity of the fractional integrodifferential problem (1.2) is a new result, and it has not been studied by other authors. Also, the global implicit function theorem was not applied to fractional problems of such a type until now.

2. Preliminaries. Let $\alpha>0$ and $h \in L^{1}\left(J, \mathbb{R}^{n}\right)$. By the left Riemann-Liouville fractional integral of $h$ on the interval $J$ we mean (cf., [21]) a function $I_{a+}^{\alpha} h$ given by:

$$
\left(I_{a+}^{\alpha} h\right)(t)=\frac{1}{\Gamma(\alpha)} \int_{a}^{t} \frac{h(\tau)}{(t-\tau)^{1-\alpha}} d \tau, \quad t \in J \text { almost everywhere }
$$

where $\Gamma$ is the Euler function. One can show that the above integral exists and is finite almost everywhere on $J$. Moreover, we also have:

Theorem 2.1. If $\alpha>0$ and $1 \leq p<\infty$, then $I_{a+}^{\alpha} h \in L^{p}\left(J, \mathbb{R}^{n}\right)$ for any $h \in L^{p}\left(J, \mathbb{R}^{n}\right)$, and

$$
\left\|I_{a+}^{\alpha} h\right\|_{L^{p}\left(J, \mathbb{R}^{n}\right)} \leq \gamma_{\alpha}\|h\|_{L^{p}\left(J, \mathbb{R}^{n}\right)}
$$

where $\gamma_{\alpha}=(b-a)^{\alpha} / \Gamma(\alpha+1)$. If, additionally, $0<\alpha<1$ and $1<p<1 / \alpha$, then $I_{a+}^{\alpha} h \in L^{q}\left(J, \mathbb{R}^{n}\right)$ for any $h \in L^{p}\left(J, \mathbb{R}^{n}\right)$, where $q=p /(1-\alpha p)$, and

$$
\left\|I_{a+}^{\alpha} h\right\|_{L^{q}\left(J, \mathbb{R}^{n}\right)} \leq \gamma_{\alpha, p, q}\|h\|_{L^{p}\left(J, \mathbb{R}^{n}\right)}
$$

for some constant $\gamma_{\alpha, p, q} \geq 0$. If $\alpha>0,1 \leq p<\infty$ and $p>1 / \alpha$, then the function $I_{a+}^{\alpha} h$ is continuous on $J$ for any $h \in L^{p}\left(J, \mathbb{R}^{n}\right)$. 
Remark 2.2. The first part of the above theorem can be found in [21]. For the second part see [9] and also [18], [21]. The last part can be deduced from the results of [9] (cf. also [21]).

Now, let $\alpha \in(0,1)$. We say that (cf., $[\mathbf{2 1}]) x \in L^{1}\left(J, \mathbb{R}^{n}\right)$ possesses the left Riemann-Liouville derivative $D_{a+}^{\alpha} x$ of order $\alpha \in(0,1)$ on the interval $J$ if the integral $I_{a+}^{1-\alpha} x$ is absolutely continuous on $J$ (more precisely, if there exists an absolutely continuous function $g$ on $J$ such that $g=I_{a+}^{1-\alpha} x$ almost everywhere on $J$ ). In such a case, we identify $I_{a+}^{1-\alpha} x$ with its absolutely continuous representant $g$ and, by the derivative $D_{a+}^{\alpha} x$, we mean the classical derivative $D^{1} g$, i.e., (after identifying $I_{a+}^{1-\alpha} x$ with $g$ )

$$
\begin{aligned}
\left(D_{a+}^{\alpha} x\right)(t) & =\frac{1}{\Gamma(1-\alpha)} D^{1}\left(\int_{a}^{t} \frac{x(\tau)}{(t-\tau)^{\alpha}} d \tau\right), \\
t & \in J \text { almost everywhere, }
\end{aligned}
$$

(cf., [12] for the case of any $\alpha>0$ ). By the value $I_{a+}^{1-\alpha} x(a)$ of the function $I_{a+}^{1-\alpha} x$ at the point $a$, we mean the value $g(a)$.

In [4], it is proved that a function $x \in L^{1}\left(J, \mathbb{R}^{n}\right)$ has the left Riemann-Liouville derivative $D_{a+}^{\alpha} x$ of order $\alpha \in(0,1)$ if and only if there exist a constant $c \in \mathbb{R}^{n}$ and a function $\varphi \in L^{1}\left(J, \mathbb{R}^{n}\right)$ such that:

$$
x(t)=\frac{1}{\Gamma(\alpha)} \frac{c}{(t-a)^{1-\alpha}}+\frac{1}{\Gamma(\alpha)} \int_{a}^{t} \frac{\varphi(\tau)}{(t-\tau)^{1-\alpha}} d \tau,
$$

for $t \in J$ almost everywhere. In such a case $I_{a+}^{1-\alpha} x(a)=c$ (after identifying $I_{a+}^{1-\alpha} x$ with its absolutely continuous representant) and $D_{a+}^{\alpha} x=\varphi$ almost everywhere on $J$. The above formula is a generalization of the well-known integral formula for absolutely continuous functions.

The set of all functions $x$ possessing Riemann-Liouville derivative $D_{a+}^{\alpha} x \in L_{n}^{2}$ is denoted by $A C_{a+}^{\alpha, 2}\left(J, \mathbb{R}^{n}\right)$. It consists of all functions $x$ possessing the representation (2.3) with $\varphi \in L_{n}^{2}$. So, if $x \in I_{a+}^{\alpha}\left(L_{n}^{2}\right)$, then $x=I_{a+}^{\alpha} D_{a+}^{\alpha} x$. Of course, the range $I_{a+}^{\alpha}\left(L_{n}^{2}\right)$ of the space $L_{n}^{2}$ under the operator $I_{a+}^{\alpha}: L_{n}^{2} \rightarrow L_{n}^{2}$ is contained in $A C_{a+}^{\alpha, 2}\left(J, \mathbb{R}^{n}\right)$. It is easy to see that $I_{a+}^{\alpha}\left(L_{n}^{2}\right)$ with the scalar product

$$
\langle x, y\rangle_{I_{a+}^{\alpha}\left(L_{n}^{2}\right)}=\left\langle D_{a+}^{\alpha} x, D_{a+}^{\alpha} y\right\rangle_{L_{n}^{2}}
$$


is complete, i.e., it is the Hilbert space. The corresponding norm in $I_{a+}^{\alpha}\left(L_{n}^{2}\right)$ is given by:

$$
\|x\|_{I_{a+}^{\alpha}\left(L_{n}^{2}\right)}=\left\|D_{a+}^{\alpha} x\right\|_{L_{n}^{2}} .
$$

3. A global implicit function theorem. Let $X$ be a real Banach space and $\varphi: X \rightarrow \mathbb{R}$ a Frechet differentiable functional. We say that $\varphi$ satisfies the Palais-Smale (PS) condition if any sequence $\left(x_{m}\right)$ such that

- $\left|\varphi\left(x_{m}\right)\right| \leq M$ for all $m \in \mathbb{N}$ and some $M>0$,

- $\varphi^{\prime}\left(x_{m}\right) \longrightarrow 0$,

admits a convergent subsequence $\left(\varphi^{\prime}\left(x_{m}\right)\right.$ denotes the Frechet differential of $\varphi$ at $\left.x_{m}\right)$. A sequence $\left(x_{m}\right)$ satisfying the above conditions is called the (PS) sequence.

In [10], the following global implicit function theorem has been derived. It is a generalization of the global inverse function theorem obtained in [14],

Theorem 3.1. Let $X$ and $Y$ be real Banach spaces, $H$ a real Hilbert space. If $F: X \times Y \rightarrow H$ is continuous differentiable with respect to $(x, y) \in X \times Y$ and

- for any $y \in Y$, the functional $\varphi_{y}: X \ni x \longmapsto \frac{1}{2}\|F(x, y)\|^{2} \in \mathbb{R}$ satisfies the (PS) condition

- $F_{x}^{\prime}(x, y): X \longrightarrow H$ is bijective for any $(x, y) \in X \times Y$,

then there exists a unique function $\lambda: Y \rightarrow X$ such that $F(\lambda(y), y)=0$ for any $y \in Y$, and this function is continuous differentiable with the differential $\lambda^{\prime}(y)$ at $y$ given by:

$$
\lambda^{\prime}(y)=-\left[F_{x}^{\prime}(\lambda(y), y)\right]^{-1} \circ F_{y}^{\prime}(\lambda(y), y) .
$$

4. The integro-differential problem. Let us consider the control system (1.2) with functions $\Phi: P_{\Delta} \times \mathbb{R}^{n} \times \mathbb{R}^{m} \rightarrow \mathbb{R}^{n}\left(P_{\Delta}=\{(t, \tau) \in\right.$ $J \times J ; \tau \leq t\}), f: J \times \mathbb{R}^{n} \times \mathbb{R}^{r} \rightarrow \mathbb{R}^{n}$ such that

(A1) $\Phi(\cdot, \cdot, x, u)$ is measurable on $P_{\Delta}$ for any $x \in \mathbb{R}^{n}, u \in \mathbb{R}^{m}$; $\Phi(t, \tau, \cdot, \cdot)$ is continuous differentiable on $\mathbb{R}^{n} \times \mathbb{R}^{m}$ for $(t, \tau) \in$ $P_{\Delta}$ almost everywhere; 
(A2) there exist functions $a_{\Phi}, b_{\Phi} \in L^{2}\left(P_{\Delta}, \mathbb{R}_{0}^{+}\right), \omega_{\Phi} \in C\left(\mathbb{R}_{0}^{+}, \mathbb{R}_{0}^{+}\right)$ and a constant $C_{\Phi}>0$ such that:

$$
\begin{gathered}
|\Phi(t, \tau, x, u)| \leq a_{\Phi}(t, \tau)|x|+b_{\Phi}(t, \tau) \omega_{\Phi}(|u|) \\
\left|\Phi_{x}(t, \tau, x, u)\right| \leq C_{\Phi}|x| \omega_{\Phi}(|u|), \\
\left|\Phi_{u}(t, \tau, x, u)\right| \leq a_{\Phi}(t, \tau)|x|+b_{\Phi}(t, \tau) \omega_{\Phi}(|u|),
\end{gathered}
$$

for $(t, \tau) \in P_{\Delta}$ almost everywhere, $x \in \mathbb{R}^{n}, u \in \mathbb{R}^{m}$;

(B1) $f(\cdot, x, u)$ is measurable on $J$ for any $x \in \mathbb{R}^{n}, v \in \mathbb{R}^{r} ; f(t, \cdot, \cdot)$ is continuous differentiable on $\mathbb{R}^{n} \times \mathbb{R}^{r}$ for $t \in J$ almost everywhere;

(B2) there exist functions $b_{f} \in L^{2}\left(J, \mathbb{R}_{0}^{+}\right), \omega_{f} \in C\left(\mathbb{R}_{0}^{+}, \mathbb{R}_{0}^{+}\right)$and constants $a_{f}, d_{f}>0$ such that:

$$
\begin{aligned}
|f(t, x, v)| & \leq a_{f}|x|+b_{f}(t) \omega_{f}(|v|) \\
\left|f_{x}(t, x, v)\right| & \leq d_{f} \omega_{f}(|v|) \\
\left|f_{v}(t, x, v)\right| & \leq a_{f}|x|+b_{f}(t) \omega_{f}(|v|)
\end{aligned}
$$

for $t \in J$ almost everywhere, $x \in \mathbb{R}^{n}, v \in \mathbb{R}^{r}$;

$(A B)$ the inequality

$$
\gamma_{\alpha}\left(\left\|a_{\Phi}\right\|_{L^{2}\left(P_{\Delta}, \mathbb{R}^{n}\right)}+a_{f}\right)<\frac{1}{\sqrt{2}}
$$

is satisfied.

We shall check that the mapping

$$
\begin{gathered}
F: I_{a+}^{\alpha}\left(L_{n}^{2}\right) \times L_{m}^{\infty} \times L_{r}^{\infty} \longrightarrow L_{n}^{2}, \\
F(x, u, v)=D_{a+}^{\alpha} x(t)+\int_{a}^{t} \Phi(t, \tau, x(\tau), u(\tau)) d \tau \\
-f(t, x(t), v(t)),
\end{gathered}
$$

satisfies assumptions of Theorem 3.1 with $X=I_{a+}^{\alpha}\left(L_{n}^{2}\right), Y=L_{m}^{\infty} \times L_{r}^{\infty}$ and $H=L_{n}^{2}$.

The well-posedness of $F$ can be checked in the same way as in [14, Proof of Lemma 5]. More precisely, in the same way as in [14, Proof of Lemma 5] one can check that the mapping

$$
I_{a+}^{\alpha}\left(L_{n}^{2}\right) \times L_{m}^{\infty} \times L_{r}^{\infty} \ni(x, u, v) \longmapsto \int_{a}^{\cdot} \Phi(\cdot, \tau, x(\tau), u(\tau)) d \tau \in L_{n}^{2}
$$


is well-posed; well-posedeness of the mapping

$$
I_{a+}^{\alpha}\left(L_{n}^{2}\right) \times L_{m}^{\infty} \times L_{r}^{\infty} \ni(x, u, v) \longmapsto D_{a+}^{\alpha} x(t)-f(t, x(t), v(t)) \in L_{n}^{2}
$$

is obvious.

Now, we shall prove:

Lemma 4.1. The operator $F$ is continuous differentiable in the Gateaux (equivalently, in the Frechet) sense on $I_{a+}^{\alpha}\left(L_{n}^{2}\right) \times L_{m}^{\infty} \times L_{r}^{\infty}$ and the mappings

$$
\begin{gathered}
F_{x}^{\prime}(x, u, v): I_{a+}^{\alpha}\left(L_{n}^{2}\right) \longrightarrow L_{n}^{2} \\
F_{x}^{\prime}(x, u, v) h=D_{a+}^{\alpha} h(t)+\int_{a}^{t} \Phi_{x}(t, \tau, x(\tau), u(\tau)) h(\tau) d \tau \\
-f_{x}(t, x(t), v(t)) h(t) \\
F_{u, v}^{\prime}(x, u, v): L_{m}^{\infty} \times L^{\infty}\left(J, \mathbb{R}^{n}\right) \rightarrow L_{n}^{2} \\
F_{u, v}^{\prime}(x, u, v)(f, g)= \\
\int_{a}^{t} \Phi_{u}(t, \tau, x(\tau), u(\tau)) f(\tau) d \tau \\
-f_{v}(t, x(t), v(t)) g(t)
\end{gathered}
$$

are the differentials of $F$ at $(x, u, v)$ in $x$ and $(u, v)$, respectively.

Proof. Let us define the auxiliary operators

$$
\begin{gathered}
P: I_{a+}^{\alpha}\left(L_{n}^{2}\right) \times L_{m}^{\infty} \times L_{r}^{\infty} \ni(x, u, v) \longmapsto D_{a+}^{\alpha} x(t) \in L_{n}^{2}, \\
Q: I_{a+}^{\alpha}\left(L_{n}^{2}\right) \times L_{m}^{\infty} \times L_{r}^{\infty} \ni(x, u, v) \longmapsto \int_{a}^{t} \Phi(t, \tau, x(\tau), u(\tau)) d \tau \in L_{n}^{2}, \\
R: I_{a+}^{\alpha}\left(L_{n}^{2}\right) \times L_{m}^{\infty} \times L_{r}^{\infty} \ni(x, u, v) \longmapsto f(t, x(t), v(t)) \in L_{n}^{2} .
\end{gathered}
$$

Of course, $F=P+Q+R$.

Differentiability of $P$. Operator $P$ is linear and continuous:

$$
\begin{aligned}
\|P(x, u, v)\|_{L_{n}^{2}} & =\left\|D_{a+}^{\alpha} x(t)\right\|_{L_{n}^{2}}=\|x\|_{I_{a+}^{\alpha}\left(L_{n}^{2}\right)} \\
& \leq\|(x, u, v)\|_{I_{a+}^{\alpha}\left(L_{n}^{2}\right) \times L_{m}^{\infty} \times L_{r}^{\infty}} .
\end{aligned}
$$

Thus, it is of class $C^{1}$. 
Differentiability of $Q$ with respect to $x$ on $I_{a+}^{\alpha}\left(L_{n}^{2}\right) \times L_{m}^{\infty} \times L_{r}^{\infty}$. Let us fix a point $(x, u, v) \in I_{a+}^{\alpha}\left(L_{n}^{2}\right) \times L_{m}^{\infty} \times L_{r}^{\infty}$. We shall show that the mapping

$$
Q_{x}^{\prime}(x, u, v): I_{a+}^{\alpha}\left(L_{n}^{2}\right) \ni h \longmapsto \int_{a}^{\cdot} \Phi_{x}(\cdot, \tau, x(\tau), u(\tau)) h(\tau) d \tau \in L_{n}^{2}
$$

is a partial Frechet differential of $Q$ with respect to $x$ at $(x, u, v)$ and $Q_{x}^{\prime}$ is continuous on $I_{a+}^{\alpha}\left(L_{n}^{2}\right) \times L_{m}^{\infty} \times L_{r}^{\infty}$. We proceed in the same way as in [15, Proof of Lemma 5]. We give here the reasoning for the convenience of the reader and because of the change of the growth condition on $\Phi_{x}$. So, if $h \in I_{a+}^{\alpha}\left(L_{n}^{2}\right) \subset L_{n}^{2}$, then the function

$$
P_{\Delta} \ni(t, \tau) \longmapsto \Phi_{x}(t, \tau, x(\tau), u(\tau)) h(\tau) \in \mathbb{R}^{n}
$$

is measurable and integrable by $(A 2)$. From the Fubini theorem, it follows that the function

$$
J \ni t \longmapsto \int_{a}^{t} \Phi_{x}(t, \tau, x(\tau), u(\tau)) h(\tau) d \tau \in \mathbb{R}^{n}
$$

is integrable. In fact,

$$
\begin{gathered}
\left|\int_{a}^{t} \Phi_{x}(t, \tau, x(\tau), u(\tau)) h(\tau) d \tau\right|^{2} \\
\leq\left(\int_{a}^{t} C_{\Phi}|x(\tau)| \omega_{\Phi}(|u(\tau)|)|h(\tau)| d \tau\right)^{2} \\
\quad \leq C_{\Phi}^{2}\left(\max \left\{\omega_{\Phi}(|u(\tau)|) ; \tau \in J\right\}\right)^{2}\left(\int_{a}^{t}|x(\tau)||h(\tau)| d \tau\right)^{2} \\
\leq C_{\Phi}^{2}\left(\max \left\{\omega_{\Phi}(|u(\tau)|) ; \tau \in J\right\}\right)^{2} \int_{a}^{b}|x(\tau)|^{2} d \tau \int_{a}^{b}|h(\tau)|^{2} d \tau
\end{gathered}
$$

So, function (4.3) belongs to $L_{n}^{2}$. It means that the operator $Q_{x}^{\prime}(x, u, v)$ is well-defined.

Clearly, $Q_{x}^{\prime}(x, u, v)$ is linear. Continuity of it follows from the following estimation:

$$
\|h\|_{L_{n}^{2}}=\left\|I_{a+}^{\alpha} D_{a+}^{\alpha} h\right\|_{L_{n}^{2}} \leq \gamma_{\alpha}\left\|D_{a+}^{\alpha} h\right\|_{L_{n}^{2}}=\gamma_{\alpha}\|h\|_{I_{a+}^{\alpha}\left(L_{n}^{2}\right)} .
$$


Now, we shall check that

$$
\lim _{\lambda \rightarrow 0} \frac{Q(x+\lambda h, u, v)-Q(x, u, v)}{\lambda}=Q_{x}^{\prime}(x, u, v) h
$$

in $L_{n}^{2}$. So, let us fix a sequence $\left(\lambda_{n}\right)$ converging to 0 in $\mathbb{R}$, and consider the limit

$$
\begin{array}{r}
\lim _{k \rightarrow \infty} \int_{a}^{b} \mid \int_{a}^{t}\left(\frac{\Phi\left(t, \tau, x(\tau)+\lambda_{k} h(\tau), u(\tau)\right)-\Phi(t, \tau, x(\tau), u(\tau))}{\lambda_{k}}\right. \\
\left.-\Phi_{x}(t, \tau, x(\tau), u(\tau)) h(\tau)\right)\left.d \tau\right|^{2} d t .
\end{array}
$$

From the differentiability of $\Phi$ with respect to $x$ we obtain, for $t \in J$ almost everywhere, the convergence of the sequence of functions

$$
\begin{gathered}
{[a, t] \ni \tau \longmapsto \frac{\Phi\left(t, \tau, x(\tau)+\lambda_{k} h(\tau), u(\tau)\right)-\Phi(t, \tau, x(\tau))}{\lambda_{k}}} \\
-\Phi_{x}(t, \tau, x(\tau), u(\tau)) h(\tau) \in \mathbb{R}^{n}
\end{gathered}
$$

almost everywhere on $[a, t]$ to the zero function. Moreover, from the mean value theorem applied to the coordinate functions

$$
[0,1] \ni \vartheta \longmapsto \Phi_{j}\left(t, \tau, x(\tau)+\vartheta \lambda_{k} h(\tau)\right) \in \mathbb{R},
$$

where $j=1, \ldots, n$, it follows that the absolute values of functions (4.4) are commonly bounded by an integrable function. Indeed, since

$$
\begin{aligned}
\Phi_{j}(t, \tau, x(\tau)+ & \left.\lambda_{k} h(\tau), u(\tau)\right)-\Phi_{j}(t, \tau, x(\tau)) \\
& =\left(\Phi_{j}\right)_{x}\left(t, \tau, x(\tau)+\vartheta_{j}(t, \tau) \lambda_{k} h(\tau) x(\tau), u(\tau)\right) \lambda_{k} h(\tau)
\end{aligned}
$$

for some $\vartheta_{j}(t, \tau) \in(0,1)$; therefore,

$$
\begin{gathered}
\left|\frac{\Phi_{j}\left(t, \tau, x(\tau)+\lambda_{k} h(\tau), u(\tau)\right)-\Phi_{j}(t, \tau, x(\tau), u(\tau))}{\lambda_{k}}\right| \\
\leq\left|\left(\Phi_{j}\right)_{x}\left(t, \tau, x(\tau)+\vartheta_{j}(t, \tau) \lambda_{k} h(\tau), u(\tau)\right)\right||h(\tau)| \\
\leq C_{\Phi}\left|x(\tau)+\vartheta_{j}(t, \tau) \lambda_{k} h(\tau)\right| \omega_{\Phi}(|u(\tau)|)|h(\tau)| \\
\leq C_{\Phi}(|x(\tau)|+|h(\tau)|) \omega_{\Phi}(|u(\tau)|)|h(\tau)|
\end{gathered}
$$

for sufficiently large $k$ (such that $\left.\left|\lambda_{k}\right|<1\right)$. Also,

$$
\left|\Phi_{x}(t, \tau, x(\tau), u(\tau)) h(\tau)\right| \leq C_{\Phi}|x(\tau)| \omega_{\Phi}(|u(\tau)|)|h(\tau)|
$$


So, using the Lebesgue dominated convergence theorem (cf., [24]) we state that the sequence of functions

$$
\begin{gathered}
J \ni t \longmapsto \int_{a}^{t}\left(\frac{\Phi\left(t, \tau, x(\tau)+\lambda_{k} h(\tau), u(\tau)\right)-\Phi(t, \tau, x(\tau), u(\tau))}{\lambda_{k}}\right. \\
\left.-\Phi_{x}(t, \tau, x(\tau), u(\tau)) h(\tau)\right) d \tau \in \mathbb{R}^{n}
\end{gathered}
$$

converges almost everywhere on $J$ to the zero function. Moreover,

$$
\begin{aligned}
& \mid \int_{a}^{t}\left(\frac{\Phi\left(t, \tau, x(\tau)+\lambda_{k} h(\tau), u(\tau)\right)-\Phi(t, \tau, x(\tau), u(\tau))}{\lambda_{k}}\right. \\
& \left.-\Phi_{x}(t, \tau, x(\tau), u(\tau)) h(\tau)\right)\left.d \tau\right|^{2} \\
& \leq\left(\int_{a}^{t} \frac{\mid \Phi\left(t, \tau, x(\tau)+\lambda_{k} h(\tau), u(\tau)\right)-\Phi(t, \tau, x(\tau), u(\tau))}{\lambda_{k}}\right. \\
& \leq\left(n \int_{a}^{t} C_{\Phi}(|x(\tau)|+|h(\tau)|) \omega_{\Phi}(|u(\tau)|)|h(\tau)| d \tau\right. \\
& \left.\quad+\int_{a}^{t} C_{\Phi}|x(\tau)| \omega_{\Phi}(|u(\tau)|)|h(\tau)| d \tau\right)^{2} \\
& \leq(n+1) \int_{a}^{b} C_{\Phi}(|x(\tau)|+|h(\tau)|) \omega_{\Phi}(|u(\tau)|)|h(\tau)| d \tau .
\end{aligned}
$$

Consequently, using once again the Lebesgue dominated convergence theorem we obtain

$$
\begin{array}{r}
\lim _{k \rightarrow \infty} \int_{a}^{b} \mid \int_{a}^{t}\left(\frac{\Phi\left(t, \tau, x(\tau)+\lambda_{k} h(\tau), u(\tau)\right)-\Phi(t, \tau, x(\tau), u(\tau))}{\lambda_{k}}\right. \\
\left.-\Phi_{x}(t, \tau, x(\tau), u(\tau)) h(\tau)\right)\left.d \tau\right|^{2} d t=0 .
\end{array}
$$

To finish this part of the proof we shall show that $Q_{x}^{\prime}$ is continuous on the space $I_{a+}^{\alpha}\left(L_{n}^{2}\right) \times L_{m}^{\infty} \times L_{r}^{\infty}$. Indeed, let $\left(x_{j}, u_{j}, v_{j}\right)$ be a sequence converging in this space to a point $\left(x_{0}, u_{0}, v_{0}\right)$. We have:

$$
\left\|\left(Q_{x}^{\prime}\left(x_{j}, u_{j}, v_{j}\right)-Q_{x}^{\prime}\left(x_{0}, u_{0}, v_{0}\right)\right) h\right\|_{L_{n}^{2}}^{2}
$$




$$
\begin{aligned}
\leq & \int_{a}^{b}\left(\int_{a}^{t} \mid \Phi_{x}\left(t, \tau, x_{j}(\tau), u_{j}(\tau)\right)\right. \\
& \left.-\Phi_{x}\left(t, \tau, x_{0}(\tau), u_{0}(\tau)\right)|| h(\tau) \mid d \tau\right)^{2} d t \\
\leq & \int_{a}^{b}\left(\int_{a}^{t}\left|\Phi_{x}\left(t, \tau, x_{j}(\tau), u_{j}(\tau)\right)-\Phi_{x}\left(t, \tau, x_{0}(\tau), u_{0}(\tau)\right)\right|^{2} d \tau\right. \\
& \left.\times \int_{a}^{t}|h(\tau)|^{2} d \tau\right) d t \\
\leq & \|h\|_{L_{n}^{2}}^{2} \int_{a}^{b} \int_{a}^{t}\left|\Phi_{x}\left(t, \tau, x_{j}(\tau), u_{j}(\tau)\right)-\Phi_{x}\left(t, \tau, x_{0}(\tau), u_{0}(\tau)\right)\right|^{2} d \tau d t \\
\leq & \left(\gamma_{\alpha}\right)^{2}\|h\|_{I_{a+}^{\alpha}\left(L_{n}^{2}\right)}^{2} \int_{a}^{b} \\
& \times \int_{a}^{t}\left|\Phi_{x}\left(t, \tau, x_{j}(\tau), u_{j}(\tau)\right)-\Phi_{x}\left(t, \tau, x_{0}(\tau), u_{0}(\tau)\right)\right|^{2} d \tau d t
\end{aligned}
$$

So,

$$
\begin{aligned}
& \left\|\left(Q_{x}^{\prime}\left(x_{j}, u_{j}, v_{j}\right)-Q_{x}^{\prime}\left(x_{0}, u_{0}, v_{0}\right)\right)\right\|_{\mathcal{L}\left(L_{n}^{2}, L_{n}^{2}\right)} \\
\leq & \gamma_{\alpha}\left(\int_{a}^{b} \int_{a}^{t}\left|\Phi_{x}\left(t, \tau, x_{j}(\tau), u_{j}(\tau)\right)-\Phi_{x}\left(t, \tau, x_{0}(\tau), u_{0}(\tau)\right)\right|^{2} d \tau d t\right)^{1 / 2}
\end{aligned}
$$

where $\mathcal{L}\left(L_{n}^{2}, L_{n}^{2}\right)$ is the space of linear continuous operators acting from $L_{n}^{2}$ to $L_{n}^{2}$, considered with the classical operator norm. From the generalized Krasnoselskii's theorem (cf., [13] with $\Omega=P_{\Delta}$ ) and the theorem on the majorized subsequence (cf., [5, Theoreme IV.9]) it follows that the above integral converges to 0 .

Differentiability of $Q$ with respect to $u$ on $I_{a+}^{\alpha}\left(L_{n}^{2}\right) \times L_{m}^{\infty} \times L_{r}^{\infty}$. Let us fix a point $(x, u, v) \in I_{a+}^{\alpha}\left(L_{n}^{2}\right) \times L_{m}^{\infty} \times L_{r}^{\infty}$ and consider the mapping

$$
Q_{u}^{\prime}(x, u, v): L_{m}^{\infty} \ni f \longmapsto \int_{a}^{\cdot} \Phi_{u}(\cdot, \tau, x(\tau), u(\tau)) f(\tau) d \tau \in L_{n}^{2}
$$

The function

$$
P_{\Delta} \ni(t, \tau) \longmapsto \Phi_{u}(t, \tau, x(\tau), u(\tau)) f(\tau)
$$

is measurable and integrable by $(A 2)$. So, the Fubini theorem implies 
integrability of the function

$$
J \ni t \longmapsto \int_{a}^{t} \Phi_{u}(t, \tau, x(\tau), u(\tau)) f(\tau) d \tau \in \mathbb{R}^{n} .
$$

Moreover,

$$
\begin{aligned}
& \left|\int_{a}^{t} \Phi_{u}(t, \tau, x(\tau), u(\tau)) f(\tau) d \tau\right|^{2} \\
& \leq\left(\|f\|_{L_{m}^{\infty}} \int_{a}^{t}\left(a_{\Phi}(t, \tau)|x(\tau)|+b_{\Phi}(t, \tau) \omega_{\Phi}(|u(\tau)|)\right) d \tau\right)^{2} \\
& \leq 2\|f\|_{L_{m}^{\infty}}^{2}\left(\int_{a}^{t} a_{\Phi}^{2}(t, \tau) d \tau \int_{a}^{t}|x(\tau)|^{2} d \tau+K_{1}^{2}\left(\int_{a}^{t} b_{\Phi}(t, \tau) d \tau\right)^{2}\right) \\
& \leq 2\|f\|_{L_{m}^{\infty}}^{2}\left(\int_{a}^{t} a_{\Phi}^{2}(t, \tau) d \tau\|x\|_{L_{n}^{2}}+K_{1}^{2}(b-a) \int_{a}^{t} b_{\Phi}^{2}(t, \tau) d \tau\right),
\end{aligned}
$$

where $K_{1}=\max \left\{\omega_{\Phi}(|u(\tau)|) ; \tau \in J\right\}$. So, in fact, the function (4.6) belongs to $L_{n}^{2}$. This means that mapping (4.5) is well defined. Of course, it is linear and continuous:

$$
\begin{aligned}
& \left(\int_{a}^{b}\left|\int_{a}^{t} \Phi_{u}(t, \tau, x(\tau), u(\tau)) f(\tau) d \tau\right|^{2} d t\right)^{1 / 2} \\
& \leq \sqrt{2}\|f\|_{L_{m}^{\infty}}\left(\|x\|_{L_{n}^{2}}^{2} \int_{a}^{b} \int_{a}^{t} a_{\Phi}^{2}(t, \tau) d \tau d t+K_{1}^{2}(b-a) \int_{a}^{b} \int_{a}^{t} b_{\Phi}^{2}(t, \tau) d \tau d t\right)^{1 / 2} \\
& =\sqrt{2}\|f\|_{L_{m}^{\infty}}\left(\|x\|_{L_{n}^{2}}^{2}\left\|a_{\Phi}\right\|_{L^{2}\left(P_{\Delta}, \mathbb{R}\right)}^{2}+K_{1}^{2}(b-a)\left\|b_{\Phi}\right\|_{L^{2}\left(P_{\Delta}, \mathbb{R}\right)}^{2}\right)^{1 / 2} .
\end{aligned}
$$

Similarly, as in the above,

$$
\lim _{\lambda \rightarrow 0} \frac{Q(x, u+\lambda f, v)-Q(x, u, v)}{\lambda}=Q_{u}^{\prime}(x, u, v) h
$$

in $L_{n}^{2}$. Indeed, let us fix a sequence $\left(\lambda_{n}\right)$ such that $\lambda_{n} \rightarrow 0$, and consider the limit

$$
\begin{array}{r}
\lim _{k \rightarrow \infty} \int_{a}^{b} \mid \int_{a}^{t}\left(\frac{\Phi\left(t, \tau, x(\tau), u(\tau)+\lambda_{k} f(\tau)\right)-\Phi(t, \tau, x(\tau), u(\tau))}{\lambda_{k}}\right. \\
\left.-\Phi_{u}(t, \tau, x(\tau), u(\tau)) f(\tau)\right)\left.d \tau\right|^{2} d t
\end{array}
$$


From the differentiability of $\Phi$ with respect to $u$ we obtain the convergence of the sequence of functions

$$
\begin{gathered}
{[a, t] \ni \tau \longmapsto \frac{\Phi\left(t, \tau, x(\tau), u(\tau)+\lambda_{k} f(\tau)\right)-\Phi(t, \tau, x(\tau), u(\tau))}{\lambda_{k}}} \\
-\Phi_{u}(t, \tau, x(\tau), u(\tau)) f(\tau) \in \mathbb{R}^{n}
\end{gathered}
$$

almost everywhere on $[0, t]$ to the zero function, for $t \in J$ almost everywhere. Moreover, from the mean value theorem applied to the coordinate functions

$$
[0,1] \ni \vartheta \longmapsto \Phi_{j}\left(t, \tau, x(\tau), u(\tau)+\vartheta \lambda_{k} f(\tau)\right) \in \mathbb{R}
$$

it follows that the absolute values of functions (4.7) are commonly bounded by an integrable function. Indeed,

$$
\begin{aligned}
& \left|\frac{\Phi_{j}\left(t, \tau, x(\tau), u(\tau)+\lambda_{k} f(\tau)\right)-\Phi_{j}(t, \tau, x(\tau), u(\tau))}{\lambda_{k}}\right| \\
& \leq\left|\left(\Phi_{j}\right)_{u}\left(t, \tau, x(\tau), u(\tau)+\vartheta_{j}(t, \tau) \lambda_{k} f(\tau)\right)\right||f(\tau)| \\
& \leq\left(a_{\Phi}(t, \tau)|x(\tau)|+b_{\Phi}(t, \tau) \omega_{\Phi}\left(\left|u(\tau)+\vartheta_{j}(t, \tau) \lambda_{k} f(\tau)\right|\right)\right)|f(\tau)| \\
& \leq\left(a_{\Phi}(t, \tau)|x(\tau)|+b_{\Phi}(t, \tau) K_{2}\right)|f(\tau)|
\end{aligned}
$$

for some $\vartheta_{j}(t, \tau) \in(0,1)$ and sufficiently large $k$ (such that $\left|\lambda_{k}\right|<1$ ), where $K_{2}=\max \left\{\omega_{\Phi}(\eta) ; 0 \leq \eta \leq\|u\|_{L_{m}^{\infty}}+\|f\|_{L_{m}^{\infty}}\right\}$. Also,

$$
\begin{aligned}
\left|\Phi_{u}(t, \tau, x(\tau), u(\tau)) f(\tau)\right| & \leq\left(a_{\Phi}(t, \tau)|x(\tau)|+b_{\Phi}(t, \tau) \omega_{\Phi}(|u(\tau)|)\right)|f(\tau)| \\
& \leq\left(a_{\Phi}(t, \tau)|x(\tau)|+b_{\Phi}(t, \tau) K_{2}\right)|f(\tau)| .
\end{aligned}
$$

So, from the Lebesgue dominated convergence theorem, it follows that, for $t \in[a, b]$ almost everywhere, the integral

$$
\begin{array}{r}
\int_{a}^{t}\left(\frac{\Phi\left(t, \tau, x(\tau), u(\tau)+\lambda_{k} f(\tau)\right)-\Phi(t, \tau, x(\tau), u(\tau))}{\lambda_{k}}\right. \\
\left.-\Phi_{u}(t, \tau, x(\tau), u(\tau)) f(\tau)\right) d \tau
\end{array}
$$

converges to 0 , i.e., the sequence of functions 


$$
\begin{array}{r}
J \ni t \longmapsto \int_{a}^{t}\left(\frac{\Phi\left(t, \tau, x(\tau), u(\tau)+\lambda_{k} f(\tau)\right)-\Phi(t, \tau, x(\tau), u(\tau))}{\lambda_{k}}\right. \\
\left.-\Phi_{u}(t, \tau, x(\tau), u(\tau)) f(\tau)\right) d \tau \in \mathbb{R}^{n}
\end{array}
$$

converges almost everywhere on $J$ to the zero function. Moreover,

$$
\begin{aligned}
\mid \int_{a}^{t}\left(\frac{\Phi\left(t, \tau, x(\tau), u(\tau)+\lambda_{k} f(\tau)\right)-\Phi(t, \tau, x(\tau), u(\tau))}{\lambda_{k}}\right. & \\
& \left.-\Phi_{u}(t, \tau, x(\tau), u(\tau)) f(\tau)\right)\left.d \tau\right|^{2} \\
\leq & \left(\int_{a}^{t} \mid \frac{\Phi\left(t, \tau, x(\tau), u(\tau)+\lambda_{k} f(\tau)\right)-\Phi(t, \tau, x(\tau), u(\tau))}{\lambda_{k}}\right. \\
& \left.-\Phi_{u}(t, \tau, x(\tau), u(\tau)) f(\tau) \mid d \tau\right)^{2} \\
\leq & \left((n+1) \int_{a}^{t}\left(a_{\Phi}(t, \tau)|x(\tau)|+b_{\Phi}(t, \tau) K_{2}\right)|f(\tau)| d \tau\right)^{2} \\
\leq & (n+1)^{2}\|f\|_{L_{m}^{\infty}}^{2} \\
& \times 2\left(\int_{a}^{t} a_{\Phi}^{2}(t, \tau) d \tau \int_{a}^{b}|x(\tau)|^{2} d \tau+K_{2}^{2}(b-a) \int_{a}^{t} b_{\Phi}^{2}(t, \tau) d \tau\right) .
\end{aligned}
$$

Consequently, from the Lebesgue dominated convergence theorem, we obtain

$$
\begin{array}{r}
\lim _{k \rightarrow \infty} \int_{a}^{b} \mid \int_{a}^{t}\left(\frac{\Phi_{j}\left(t, \tau, x(\tau), u(\tau)+\lambda_{k} f(\tau)\right)-\Phi(t, \tau, x(\tau), u(\tau))}{\lambda_{k}}\right. \\
\left.-\Phi_{x}(t, \tau, x(\tau), u(\tau)) f(\tau)\right)\left.d \tau\right|^{2} d t=0 .
\end{array}
$$

Now, we shall show that $Q_{u}^{\prime}$ is continuous on the space

$$
I_{a+}^{\alpha}\left(L_{n}^{2}\right) \times L_{m}^{\infty} \times L_{r}^{\infty} .
$$

Indeed, let $\left(x_{j}, u_{j}, v_{j}\right)$ be a sequence converging in this space to a point $\left(x_{0}, u_{0}, v_{0}\right)$. We have:

$$
\left\|\left(Q_{u}^{\prime}\left(x_{j}, u_{j}, v_{j}\right)-Q_{u}^{\prime}\left(x_{0}, u_{0}, v_{0}\right)\right) f\right\|_{L_{n}^{2}}^{2}
$$




$$
\begin{aligned}
& \leq \int_{a}^{b}\left(\int_{a}^{t}\left|\Phi_{u}\left(t, \tau, x_{j}(\tau), u_{j}(\tau)\right)-\Phi_{u}\left(t, \tau, x_{0}(\tau), u_{0}(\tau)\right)\right||f(\tau)| d \tau\right)^{2} d t \\
& \leq\|f\|_{L_{m}^{\infty}}^{2} \int_{a}^{b}\left(\int_{a}^{t}\left|\Phi_{u}\left(t, \tau, x_{j}(\tau), u_{j}(\tau)\right)-\Phi_{u}\left(t, \tau, x_{0}(\tau), u_{0}(\tau)\right)\right| d \tau\right)^{2} d t
\end{aligned}
$$

Thus,

$$
\begin{aligned}
& \left\|Q_{u}^{\prime}\left(x_{j}, u_{j}, v_{j}\right)-Q_{u}^{\prime}\left(x_{0}, u_{0}, v_{0}\right)\right\|_{\mathcal{L}\left(L_{m}^{\infty}, L_{n}^{2}\right)} \\
& \leq\left(\int_{a}^{b}\left(\int_{a}^{t}\left|\Phi_{u}\left(t, \tau, x_{j}(\tau), u_{j}(\tau)\right)-\Phi_{u}\left(t, \tau, x_{0}(\tau), u_{0}(\tau)\right)\right| d \tau\right)^{2} d t\right)^{1 / 2} .
\end{aligned}
$$

Of course, there exists a subsequence $\left(x_{j_{i}}, u_{j_{i}}, v_{j_{i}}\right)$ such that $x_{j_{i}} \rightarrow x_{0}$ and $u_{j_{i}} \rightarrow u_{0}$ pointwise almost everywhere on $J$. So, for $t \in J$ almost everywhere, the sequence of functions

$$
[a, t] \ni \tau \longmapsto\left|\Phi_{u}\left(t, \tau, x_{j_{i}}(\tau), u_{j}(\tau)\right)-\Phi_{u}\left(t, \tau, x_{0}(\tau), u_{0}(\tau)\right)\right| \in \mathbb{R}
$$

is pointwise converging to zero function and majorized by an integrable function:

$$
\begin{aligned}
& \left|\Phi_{u}\left(t, \tau, x_{j_{i}}(\tau), u_{j_{i}}(\tau)\right)-\Phi_{u}\left(t, \tau, x_{0}(\tau), u_{0}(\tau)\right)\right| \\
\leq & a_{\Phi}(t, \tau)\left|x_{j_{i}}(\tau)\right|+b_{\Phi}(t, \tau) \omega_{\Phi}\left(\left|u_{j_{i}}(\tau)\right|\right) \\
& +a_{\Phi}(t, \tau)\left|x_{0}(\tau)\right|+b_{\Phi}(t, \tau) \omega_{\Phi}\left(\left|u_{0}(\tau)\right|\right) \\
\leq & a_{\Phi}(t, \tau)\left|x_{j_{i}}(\tau)\right|+2 b_{\Phi}(t, \tau) K_{3}+a_{\Phi}(t, \tau)\left|x_{0}(\tau)\right|,
\end{aligned}
$$

where $K_{3}=\max \left\{\omega_{\Phi}\left(\left|u_{j}(\tau)\right|\right) ; \tau \in J, j=0,1, \ldots\right\}$. So, the sequence of functions

$$
J \ni t \longmapsto \int_{a}^{t}\left|\Phi_{u}\left(t, \tau, x_{j_{i}}(\tau), u_{j_{i}}(\tau)\right)-\Phi_{u}\left(t, \tau, x_{0}(\tau), u_{0}(\tau)\right)\right| d \tau \in \mathbb{R}
$$

converges pointwise to the zero function. Moreover,

$$
\begin{aligned}
& \left(\int_{a}^{t}\left|\Phi_{u}\left(t, \tau, x_{j_{i}}(\tau), u_{j}(\tau)\right)-\Phi_{u}\left(t, \tau, x_{0}(\tau), u_{0}(\tau)\right)\right| d \tau\right)^{2} \\
& \leq\left(\int_{a}^{t}\left(\left|\Phi_{u}\left(t, \tau, x_{j_{i}}(\tau), u_{j}(\tau)\right)\right|+\left|\Phi_{u}\left(t, \tau, x_{0}(\tau), u_{0}(\tau)\right)\right|\right) d \tau\right)^{2} \\
& \leq\left(\int _ { a } ^ { t } \left(a_{\Phi}(t, \tau)\left|x_{j_{i}}(\tau)\right|+b_{\Phi}(t, \tau) \omega_{\Phi}\left(\left|u_{j_{i}}(\tau)\right|\right)\right.\right.
\end{aligned}
$$




$$
\begin{gathered}
\left.\left.+a_{\Phi}(t, \tau)\left|x_{0}(\tau)\right|+b_{\Phi}(t, \tau) \omega_{\Phi}\left(\left|u_{0}(\tau)\right|\right)\right) d \tau\right)^{2} \\
\leq\left(\left(\int_{a}^{t} a_{\Phi}^{2}(t, \tau) d \tau\right)^{1 / 2}\left(\int_{a}^{t}\left|x_{j_{i}}(\tau)\right|^{2} d \tau\right)^{1 / 2}+2 K_{3} \int_{a}^{t} b_{\Phi}(t, \tau) d \tau\right. \\
\left.\quad+\left(\int_{a}^{t} a_{\Phi}^{2}(t, \tau) d \tau\right)^{1 / 2}\left(\int_{a}^{t}\left|x_{0}(\tau)\right|^{2} d \tau\right)^{1 / 2}\right)^{2} \\
\leq\left(\left(2 \int_{a}^{t} a_{\Phi}^{2}(t, \tau) d \tau\right)^{1 / 2} \gamma_{\alpha} M+2 K_{3} \sqrt{b-a}\left(\int_{a}^{t} b_{\Phi}^{2}(t, \tau) d \tau\right)^{1 / 2}\right)^{2}
\end{gathered}
$$

where $M>0$ is such that $\left\|x_{j}\right\|_{I_{a+}^{\alpha}\left(L_{n}^{2}\right)} \leq M$ for $j=0,1, \ldots$ From the classical Lebesgue theorem on the dominated convergence it follows that

$$
\left\|Q_{u}^{\prime}\left(x_{j_{i}}, u_{j_{i}}, v_{j_{i}}\right)-Q_{u}^{\prime}\left(x_{0}, u_{0}, v_{0}\right)\right\|_{\mathcal{L}\left(L_{m}^{\infty}, L_{n}^{2}\right)} \longrightarrow 0 .
$$

In fact this means that

$$
\left\|Q_{u}^{\prime}\left(x_{j}, u_{j}, v_{j}\right)-Q_{u}^{\prime}\left(x_{0}, u_{0}, v_{0}\right)\right\|_{\mathcal{L}\left(L_{m}^{\infty}, L_{n}^{2}\right)} \longrightarrow 0 .
$$

Indeed, in the opposite case, we could choose a subsequence such that

$$
0<\varepsilon<\left\|Q_{u}^{\prime}\left(x_{j_{k}}, u_{j_{k}}, v_{j_{k}}\right)-Q_{u}^{\prime}\left(x_{0}, u_{0}, v_{0}\right)\right\|_{\mathcal{L}\left(L_{m}^{\infty}, L_{n}^{2}\right)}
$$

for $k \in \mathbb{N}$ and some $\varepsilon>0$. Repeating the above reasoning we could choose a subsequence $\left(x_{j_{k_{i}}}, u_{j_{k_{i}}}, v_{j_{k_{i}}}\right)$ such that

$$
\left\|Q_{u}^{\prime}\left(x_{j_{k_{i}}}, u_{j_{k_{i}}}, v_{j_{k_{i}}}\right)-Q_{u}^{\prime}\left(x_{0}, u_{0}, v_{0}\right)\right\|_{\mathcal{L}\left(L_{m}^{\infty}, L_{n}^{2}\right)} \longrightarrow 0
$$

in contrast to (4.8).

Differentiability of $R$ with respect to $x$ on $I_{a+}^{\alpha}\left(L_{n}^{2}\right) \times L_{m}^{\infty} \times L_{r}^{\infty}$. Let us fix a point $(x, u, v) \in I_{a+}^{\alpha}\left(L_{n}^{2}\right) \times L_{m}^{\infty} \times L_{r}^{\infty}$. It is easy to see that the mapping

$$
R_{x}^{\prime}(x, u, v): I_{a+}^{\alpha}\left(L_{n}^{2}\right) \ni h \longmapsto f_{x}(\cdot, x(\cdot), v(\cdot)) h(\cdot) \in L_{n}^{2}
$$

is well-defined, linear and continuous:

$$
\begin{aligned}
\int_{a}^{b}\left|f_{x}(t, x(t), v(t)) h(t)\right|^{2} d t & \leq \int_{a}^{b}\left|f_{x}(t, x(t), v(t))\right|^{2}|h(t)|^{2} d t \\
& \leq \int_{a}^{b}\left(d_{f}^{2}\left(\omega_{f}(|v(t)|)\right)^{2}|h(t)|^{2} d t\right.
\end{aligned}
$$




$$
\leq d_{f}^{2} K_{4}^{2}\|h\|_{L_{n}^{2}}^{2} \leq d_{f}^{2} K_{4}^{2}\left(\gamma_{\alpha}\right)^{2}\|h\|_{I_{a+}^{\alpha}\left(L_{n}^{2}\right)}^{2},
$$

where $K_{4}=\max \left\{\omega_{f}(|v(t)| ; t \in J\}\right.$. In the same way as in the previous cases we check that $R_{x}^{\prime}(x, u, v)$ is the differential of $R$ at $(x, u, v)$ with respect to $x$. First we check that

$$
\begin{aligned}
\lim _{k \rightarrow \infty} \int_{a}^{b} \mid \frac{f\left(t, x(t)+\lambda_{k} h(t), v(t)\right)-f(t, x(t), v(t))}{\lambda_{k}} & \\
& \left.-f_{x}(t, x(t), v(t)) h(t)\right)\left.\right|^{2} d t=0 .
\end{aligned}
$$

Indeed, it is clear that the sequence of the above integrands converges pointwise to the zero function. Basing this on the mean value theorem we assert that this sequence is majorized by an integrable function, namely, $(n+1)^{2} d_{f}^{2} K_{4}^{2}|h(\cdot)|^{2}$. So, it is sufficient to use the Lebesgue dominated convergence theorem.

To finish this part of the proof we shall demonstrate that the mapping

$$
R^{\prime}: I_{a+}^{\alpha}\left(L_{n}^{2}\right) \times L_{m}^{\infty} \times L_{r}^{\infty} \ni(x, u, v) \longmapsto R_{x}^{\prime}(x, u, v) \in \mathcal{L}\left(I_{a+}^{\alpha}\left(L_{n}^{2}\right), L_{n}^{2}\right)
$$

is continuous. So, let $\left(x_{j}, u_{j}, v_{j}\right)$ be a sequence converging in $I_{a+}^{\alpha}\left(L_{n}^{2}\right) \times$ $L_{m}^{\infty} \times L_{r}^{\infty}$ to a point $\left(x_{0}, u_{0}, v_{0}\right)$. Similarly, as in the previous case, we may assume that $x_{j} \rightarrow x_{0}$ and $v_{j} \rightarrow v_{0}$ pointwise almost everywhere on $J$. We have:

$$
\begin{aligned}
& \left\|\left(R_{x}^{\prime}\left(x_{j}, u_{j}, v_{j}\right)-R_{x}^{\prime}\left(x_{0}, u_{0}, v_{0}\right)\right) h\right\|_{L_{n}^{2}}^{2} \\
& =\int_{a}^{b}\left|\left(f_{x}\left(t, x_{j}(t), v_{j}(t)\right)-f_{x}\left(t, x_{0}(t), v_{0}(t)\right)\right) h(t)\right|^{2} d t \\
& \leq \int_{a}^{b}\left|f_{x}\left(t, x_{j}(t), v_{j}(t)\right)-f_{x}\left(t, x_{0}(t), v_{0}(t)\right)\right|^{2}|h(t)|^{2} d t .
\end{aligned}
$$

Now, we shall consider three cases. The reasoning that we present below is due to Kamocki, and it is contained in [17].

Case $1^{0}$. Assume that $1 / 2<\alpha$. Then

$$
|h(t)|=\left|I_{a+}^{\alpha}\left(D_{a+}^{\alpha} h\right)(t)\right| \leq \frac{1}{\Gamma(\alpha)} \int_{a}^{t} \frac{\left|D_{a+}^{\alpha} h(\tau)\right|}{(t-\tau)^{1-\alpha}} d \tau
$$




$$
\begin{aligned}
& \leq \frac{1}{\Gamma(\alpha)}\left\|D_{a+}^{\alpha} h\right\|_{L_{n}^{2}} \int_{a}^{t} \frac{d \tau}{(t-\tau)^{(1-\alpha) 2}} \\
& =\frac{1}{\Gamma(\alpha)}\left\|D_{a+}^{\alpha} h\right\|_{L_{n}^{2}} \frac{1}{2 \alpha-1}(t-a)^{2 \alpha-1} \\
& \leq \frac{1}{\Gamma(\alpha)}\left\|D_{a+}^{\alpha} h\right\|_{L_{n}^{2}} \frac{1}{2 \alpha-1}(b-a)^{2 \alpha-1} \\
& =\frac{1}{\Gamma(\alpha)} \frac{1}{2 \alpha-1}(b-a)^{2 \alpha-1}\|h\|_{I_{a+}^{\alpha}\left(L_{n}^{2}\right)}
\end{aligned}
$$

for $t \in J$ almost everywhere. So, continuing (4.9), we obtain

$$
\begin{aligned}
& \int_{a}^{b}\left|f_{x}\left(t, x_{j}(t), v_{j}(t)\right)-f_{x}\left(t, x_{0}(t), v_{0}(t)\right)\right|^{2}|h(t)|^{2} d t \\
& \leq\left(\frac{1}{\Gamma(\alpha)} \frac{1}{2 \alpha-1}(b-a)^{2 \alpha-1}\right)^{2}\|h\|_{I_{a+}^{\alpha}\left(L_{n}^{2}\right)}^{2} \\
& \quad \times \int_{a}^{b}\left|f_{x}\left(t, x_{j}(t), v_{j}(t)\right)-f_{x}\left(t, x_{0}(t), v_{0}(t)\right)\right|^{2} d t .
\end{aligned}
$$

Thus, in this case,

$$
\begin{aligned}
& \left\|R_{x}^{\prime}\left(x_{j}, u_{j}, v_{j}\right)-R_{x}^{\prime}\left(x_{0}, u_{0}, v_{0}\right)\right\|_{\mathcal{L}\left(I_{a+}^{\alpha}\left(L_{n}^{2}\right), L_{n}^{2}\right)} \\
& \leq \frac{1}{\Gamma(\alpha)} \frac{1}{2 \alpha-1}(b-a)^{2 \alpha-1} \\
& \quad \times\left(\int_{a}^{b}\left|f_{x}\left(t, x_{j}(t), v_{j}(t)\right)-f_{x}\left(t, x_{0}(t), v_{0}(t)\right)\right|^{2} d t\right)^{1 / 2} .
\end{aligned}
$$

Convergence of the integral $\int_{a}^{b}\left|f_{x}\left(t, x_{j}(t), v_{j}(t)\right)-f_{x}\left(t, x_{0}(t), v_{0}(t)\right)\right|^{2} d t$ to 0 follows from the Lebesgue dominated convergence theorem (the integrands are bounded almost everywhere on $[a, b]$ by a constant (cf., $(B 2)))$.

Case $2^{0}$. Assume that $0<\alpha<1 / 2$. Then, from the second part of Theorem 2.1, it follows that if $h \in I_{a+}^{\alpha}\left(L_{n}^{2}\right)$, then $h \in L_{n}^{2 /(1-2 \alpha)}$ and, consequently, $|h|^{2} \in L_{1}^{q}$ with $q=1 /(1-2 \alpha)$ (of course, $1<$ $1 /(1-2 \alpha)<\infty)$. Function $\left|f_{x}\left(\cdot, x_{j}(\cdot), v_{j}(\cdot)\right)-f_{x}\left(\cdot, x_{0}(\cdot), v_{0}(\cdot)\right)\right|^{2}$ being essentially bounded belongs to $L_{1}^{q^{\prime}}$ with $q^{\prime}=q /(q-1)$. Consequently,

$$
\int_{a}^{b}\left|f_{x}\left(t, x_{j}(t), v_{j}(t)\right)-f_{x}\left(t, x_{0}(t), v_{0}(t)\right)\right|^{2}|h(t)|^{2} d t
$$




$$
\begin{aligned}
\leq & \left(\int_{a}^{b}\left|f_{x}\left(t, x_{j}(t), v_{j}(t)\right)-f_{x}\left(t, x_{0}(t), v_{0}(t)\right)\right|^{2 q^{\prime}} d t\right)^{1 / q^{\prime}} \\
& \times\left(\int_{a}^{b}|h(t)|^{2 q} d t\right)^{1 / q} \\
= & \left\|f_{x}\left(\cdot, x_{j}(\cdot), v_{j}(\cdot)\right)-f_{x}\left(\cdot, x_{0}(\cdot), v_{0}(\cdot)\right) \mid\right\|_{L_{1}^{2 q^{\prime}}}^{2}\|h\|_{L_{n}^{2 q}}^{2} \\
= & \left\|f_{x}\left(\cdot, x_{j}(\cdot), v_{j}(\cdot)\right)-f_{x}\left(\cdot, x_{0}(\cdot), v_{0}(\cdot)\right) \mid\right\|_{L_{1}^{2 q^{\prime}}}^{2}\left\|I_{a+}^{\alpha} D_{a+}^{\alpha} h\right\|_{L_{n}^{2 q}}^{2} \\
\leq & \left\|f_{x}\left(\cdot, x_{j}(\cdot), v_{j}(\cdot)\right)-f_{x}\left(\cdot, x_{0}(\cdot), v_{0}(\cdot)\right) \mid\right\|_{L_{1}^{2 q^{\prime}}}^{2} \gamma_{\alpha, 2,2 q}^{2}\left\|D_{a+}^{\alpha} h\right\|_{L_{n}^{2}}^{2} \\
= & \left\|f_{x}\left(\cdot, x_{j}(\cdot), v_{j}(\cdot)\right)-f_{x}\left(\cdot, x_{0}(\cdot), v_{0}(\cdot)\right) \mid\right\|_{L_{1}^{2 q^{\prime}}}^{2} \gamma_{\alpha, 2,2 q}^{2}\|h\|_{I_{a+}^{\alpha}\left(L_{n}^{2}\right)}^{2} .
\end{aligned}
$$

So,

$$
\begin{aligned}
& \left\|R_{x}^{\prime}\left(x_{j}, u_{j}, v_{j}\right)-R_{x}^{\prime}\left(x_{0}, u_{0}, v_{0}\right)\right\|_{\mathcal{L}\left(I_{a+}^{\alpha}\left(L_{n}^{2}\right), L_{n}^{2}\right)} \\
& \quad \leq\left\|\mid f_{x}\left(\cdot, x_{j}(\cdot), v_{j}(\cdot)\right)-f_{x}\left(\cdot, x_{0}(\cdot), v_{0}(\cdot)\right)\right\|_{L_{1}^{2 q^{\prime}}} \gamma_{\alpha, 2,2 q} .
\end{aligned}
$$

In the same way as in case $1^{0}$, convergence

$$
\int_{a}^{b}\left|f_{x}\left(t, x_{j}(t), v_{j}(t)\right)-f_{x}\left(t, x_{0}(t), v_{0}(t)\right)\right|^{2 q^{\prime}} d t \longrightarrow 0
$$

follows from the Lebesgue dominated convergence theorem.

Case $3^{0}$. Assume that $\alpha=1 / 2$. Let us observe that, if $z \in L_{n}^{2}$, then $z \in L^{p}\left(J, \mathbb{R}^{n}\right)$ for any $p \in(1,2)$ and (cf., the second part of Theorem 2.1) $I_{a+}^{\alpha} z \in L^{q}\left(J, \mathbb{R}^{n}\right)$ where

$$
q=\frac{p}{1-\alpha p}=\frac{2 p}{2-p}>2
$$

as well as

$$
\begin{aligned}
\left\|I_{a+}^{\alpha} z\right\|_{L^{q}\left(J, \mathbb{R}^{n}\right)} & \leq c_{\alpha, p, q}\|z\|_{L^{p}\left(J, \mathbb{R}^{n}\right)} \\
& \leq \gamma_{\alpha, p, q}\left(\left(\int_{a}^{b} 1 d t\right)^{1-p / 2}\left(\int_{a}^{b}\left(|z(t)|^{p}\right)^{2 / p} d t\right)^{p / 2}\right)^{1 / p} \\
& =\gamma_{\alpha, p, q}(b-a)^{(2-p) / 2 p}\|z\|_{L_{n}^{2}} .
\end{aligned}
$$

So, for arbitrary fixed $p \in(1,2)$,

$$
q=\frac{p}{1-\alpha p}, \quad q^{\prime}=\frac{q}{q-2},
$$


we obtain

$$
\begin{aligned}
& \int_{a}^{b}\left|f_{x}\left(t, x_{j}(t), v_{j}(t)\right)-f_{x}\left(t, x_{0}(t), v_{0}(t)\right)\right|^{2}|h(t)|^{2} d t \\
&=\int_{a}^{b}\left|f_{x}\left(t, x_{j}(t), v_{j}(t)\right)-f_{x}\left(t, x_{0}(t), v_{0}(t)\right)\right|^{2}\left|I_{a+}^{\alpha} D_{a+}^{\alpha} h(t)\right|^{2} d t \\
& \leq\left(\int_{a}^{b}\left|f_{x}\left(t, x_{j}(t), v_{j}(t)\right)-f_{x}\left(t, x_{0}(t), v_{0}(t)\right)\right|^{2 q^{\prime}} d t\right)^{1 / q^{\prime}} \\
& \quad \times\left(\int_{a}^{b}\left(\left|I_{a+}^{\alpha} D_{a+}^{\alpha} h(t)\right|^{2}\right)^{q / 2} d t\right)^{2 / q} \\
&=\left\|\left|f_{x}\left(\cdot, x_{j}(\cdot), v_{j}(\cdot)\right)-f_{x}\left(\cdot, x_{0}(\cdot), v_{0}(\cdot)\right)\right|\right\|_{L_{1}^{2 q^{\prime}}}^{2}\left\|I_{a+}^{\alpha} D_{a+}^{\alpha} h\right\|_{L_{n}^{q}}^{2} \\
& \leq\left\|\left|f_{x}\left(\cdot, x_{j}(\cdot), v_{j}(\cdot)\right)-f_{x}\left(\cdot, x_{0}(\cdot), v_{0}(\cdot)\right)\right|\right\|_{L_{1}^{2 q^{\prime}}}^{2} \\
& \times \gamma_{\alpha, p, q}^{2}(b-a)^{(2-p) / p}\left\|D_{a+}^{\alpha} h\right\|_{L_{n}^{2}}^{2} \\
&=\left\|\left|f_{x}\left(\cdot, x_{j}(\cdot), v_{j}(\cdot)\right)-f_{x}\left(\cdot, x_{0}(\cdot), v_{0}(\cdot)\right)\right|\right\|_{L_{1}^{2 q^{\prime}}}^{2} \\
& \times \gamma_{\alpha, p, q}^{2}(b-a)^{(2-p) / p}\|h\|_{I_{a+}^{\alpha}\left(L_{n}^{2}\right)}^{2} .
\end{aligned}
$$

Similarly, as in the previous cases, convergence

$$
\int_{a}^{b}\left|f_{x}\left(t, x_{j}(t), v_{j}(t)\right)-f_{x}\left(t, x_{0}(t), v_{0}(t)\right)\right|^{2 q^{\prime}} d t \longrightarrow 0
$$

follows from the Lebesgue dominated convergence theorem.

Differentiability of $R$ with respect to $v$ on $I_{a+}^{\alpha}\left(L_{n}^{2}\right) \times L_{m}^{\infty} \times L_{r}^{\infty}$. Let us fix a point $(x, u, v) \in I_{a+}^{\alpha}\left(L_{n}^{2}\right) \times L_{m}^{\infty} \times L_{r}^{\infty}$. Clearly, the mapping

$$
R_{v}^{\prime}(x, u, v): L_{r}^{\infty} \ni g \longmapsto f_{v}(\cdot, x(\cdot), v(\cdot)) g(\cdot) \in L_{n}^{2}
$$

is well defined, linear and continuous:

$$
\begin{aligned}
& \int_{a}^{b}\left|f_{v}(t, x(t), v(t)) g(t)\right|^{2} d t \\
& \leq \int_{a}^{b}\left|f_{v}(t, x(t), v(t))\right|^{2}|g(t)|^{2} d t \\
& \leq \int_{a}^{b}\left(a_{f}|x(t)|+b_{f}(t) \omega_{f}(|v(t)|)\right)^{2}|g(t)|^{2} d t
\end{aligned}
$$




$$
\leq\|g\|_{L_{r}^{\infty}}^{2} \int_{a}^{b}\left(a_{f}|x(t)|+b_{f}(t) \omega_{f}(|v(t)|)\right)^{2} d t .
$$

In the same way as in the previous cases, using the Lebesgue dominated convergence theorem, we check that

$$
\begin{aligned}
\lim _{k \rightarrow \infty} \int_{a}^{b} \mid \frac{f\left(t, x(t), v(t)+\lambda_{k} g(t)\right)-f(t, x(t), v(t))}{\lambda_{k}} & \\
& \left.-f_{v}(t, x(t), v(t)) g(t)\right)\left.\right|^{2} d t=0 .
\end{aligned}
$$

Moreover, the mapping

$$
R^{\prime}: I_{a+}^{\alpha}\left(L_{n}^{2}\right) \times L_{m}^{\infty} \times L_{r}^{\infty} \ni(x, u, v) \longmapsto R_{x}^{\prime}(x, u, v) \in \mathcal{L}\left(L_{r}^{\infty}, L_{n}^{2}\right)
$$

is continuous. Indeed, let $\left(x_{j}, u_{j}, v_{j}\right)$ be a sequence converging in $I_{a+}^{\alpha}\left(L_{n}^{2}\right) \times L_{m}^{\infty} \times L_{r}^{\infty}$ to a point $\left(x_{0}, u_{0}, v_{0}\right)$. As in the previous cases, we may assume that $x_{j} \rightarrow x_{0}$ and $v_{j} \rightarrow v_{0}$ pointwise almost everywhere on $J$. We have

$$
\begin{aligned}
& \left\|\left(R_{v}^{\prime}\left(x_{j}, u_{j}, v_{j}\right)-R_{v}^{\prime}\left(x_{0}, u_{0}, v_{0}\right)\right)\right\|_{L_{n}^{2}}^{2} \\
& =\int_{a}^{b}\left|\left(f_{v}\left(t, x_{j}(t), v_{j}(t)\right)-f_{v}\left(t, x_{0}(t), v_{0}(t)\right)\right) g(t)\right|^{2} d t \\
& \leq \int_{a}^{b}\left|f_{v}\left(t, x_{j}(t), v_{j}(t)\right)-f_{v}\left(t, x_{0}(t), v_{0}(t)\right)\right|^{2}|g(t)|^{2} d t \\
& \leq\|g\|_{L_{r}^{\infty}}^{2} \int_{a}^{b}\left|f_{v}\left(t, x_{j}(t), v_{j}(t)\right)-f_{v}\left(t, x_{0}(t), v_{0}(t)\right)\right|^{2} d t .
\end{aligned}
$$

So,

$$
\begin{aligned}
& \left\|\left(R_{v}^{\prime}\left(x_{j}, u_{j}, v_{j}\right)-R_{v}^{\prime}\left(x_{0}, u_{0}, v_{0}\right)\right) g\right\|_{\mathcal{L}\left(L_{r}^{\infty}, L_{n}^{2}\right)} \\
& \quad \leq\left(\int_{a}^{b}\left|f_{v}\left(t, x_{j}(t), v_{j}(t)\right)-f_{v}\left(t, x_{0}(t), v_{0}(t)\right)\right|^{2} d t\right)^{1 / 2} .
\end{aligned}
$$

Using once again the Lebesgue dominated convergence theorem, we assert that

$$
\int_{a}^{b}\left|f_{v}\left(t, x_{j}(t), v_{j}(t)\right)-f_{v}\left(t, x_{0}(t), v_{0}(t)\right)\right|^{2} d t \longrightarrow 0 .
$$

The proof is completed. 
Lemma 4.2. For any fixed $(x, u, v) \in I_{a+}^{\alpha}\left(L_{n}^{2}\right) \times L_{m}^{\infty} \times L_{r}^{\infty}$, the operator $F_{x}^{\prime}(x, u, v): I_{a+}^{\alpha}\left(L_{n}^{2}\right) \rightarrow L_{n}^{2}$ given by (4.2) is "one to one" and "onto."

Proof. Let us fix a function $z \in L_{n}^{2}$ and consider in $I_{a+}^{\alpha}\left(L_{n}^{2}\right)$ the equation

$$
D_{a+}^{\alpha} h(t)+\int_{a}^{t} \Phi_{x}(t, \tau, x(\tau), u(\tau)) h(\tau) d \tau-f_{x}(t, x(t), v(t)) h(t)=z(t) .
$$

Putting

$$
\begin{aligned}
\Psi(t, \tau, h) & =\Phi_{x}(t, \tau, x(\tau), u(\tau)) h \\
g(t, h) & =f_{x}(t, x(t), v(t)) h+z(t),
\end{aligned}
$$

we see that $\Psi$ and $g$ satisfy assumptions from the Appendix with

$$
d(t, \tau)=C_{\Phi}|x(\tau)| \omega_{\Phi}(|u(\tau)|)
$$

and

$$
L=d_{f} \max \left\{\omega_{f}(|v(\tau)|) ; \tau \in J\right\}
$$

So, from Lemma 6.1 and the observation formulated before this lemma it follows that equation (4.10) has a unique solution in $I_{a+}^{\alpha}\left(L_{n}^{2}\right)$. The proof is completed.

Now, let us fix a function $(u, v) \in L_{m}^{\infty} \times L_{r}^{\infty}$ and consider the functional

$$
\begin{aligned}
& \varphi: I_{a+}^{\alpha}\left(L_{n}^{2}\right) \ni x \longmapsto \frac{1}{2}\|F(x, u, v)\|^{2} \\
= & \frac{1}{2} \int_{a}^{b}\left|D_{a+}^{\alpha} x(t)+\int_{a}^{t} \Phi(t, \tau, x(\tau), u(\tau)) d \tau-f(t, x(t), v(t))\right|^{2} d t \in \mathbb{R} .
\end{aligned}
$$

It is easy to see that, for any $x \in I_{a+}^{\alpha}\left(L_{n}^{2}\right)$,

$$
\begin{aligned}
|\varphi(x)|^{1 / 2} \geq & \frac{1}{\sqrt{2}}\left(\|x\|_{I_{a+}^{\alpha}\left(L_{n}^{2}\right)}\right. \\
& \left.-\left\|\int_{a}^{\cdot} \Phi(\cdot, \tau, x(\tau), u(\tau)) d \tau\right\|_{L_{n}^{2}}-\|f(\cdot, x(\cdot), u(\cdot))\|_{L_{n}^{2}}\right) .
\end{aligned}
$$


Moreover,

$$
\begin{aligned}
& \left(\int_{a}^{b}\left|\int_{a}^{t} \Phi(t, \tau, x(\tau), u(\tau)) d \tau\right|^{2} d t\right)^{1 / 2} \\
& \leq\left(\int_{a}^{b}\left(\int_{a}^{t}\left(a_{\Phi}(t, \tau)|x(\tau)|+b_{\Phi}(t, \tau) \omega_{\Phi}(|u(\tau)|)\right) d \tau\right)^{2} d t\right)^{1 / 2} \\
& \leq\left(\int_{a}^{b}\left(\int_{a}^{t} a_{\Phi}^{2}(t, \tau) d \tau\right)^{1 / 2}\left(\int_{a}^{t}|x(\tau)|^{2} d \tau\right)^{1 / 2}\right. \\
& \left.\left.\quad+K_{1} \sqrt{b-a}\left(\int_{a}^{t} b_{\Phi}^{2}(t, \tau) d \tau\right)^{1 / 2}\right)^{2} d t\right)^{1 / 2} \\
& \leq \sqrt{2}\left(\int _ { a } ^ { b } \left(\left(\int_{a}^{t} a_{\Phi}^{2}(t, \tau) d \tau\right)\left(\int_{a}^{t}|x(\tau)|^{2} d \tau\right)+K_{1}^{2}(b-a)\right.\right. \\
& \left.\left.\left.\quad \times\left(\int_{a}^{t} b_{\Phi}^{2}(t, \tau) d \tau\right)\right) d t\right)^{1 / 2}\right)^{\leq} \sqrt{2}\left(\|x\|_{L_{n}^{2}}^{2}\left\|a_{\Phi}\right\|_{L^{2}\left(P_{\Delta}, \mathbb{R}\right)}^{2}+K_{1}^{2}(b-a)\left\|b_{\Phi}\right\|_{L^{2}\left(P_{\Delta}, \mathbb{R}\right)}^{2}\right)^{1 / 2} \\
& \leq \sqrt{2}\left(\|x\|_{L_{n}^{2}}\left\|a_{\Phi}\right\|_{L^{2}\left(P_{\Delta}, \mathbb{R}\right)}+K_{1} \sqrt{b-a}\left\|b_{\Phi}\right\|_{L^{2}\left(P_{\Delta}, \mathbb{R}\right)}\right) \\
& \leq \sqrt{2}\left(\gamma_{\alpha}\|x\|_{I_{a+}^{\alpha}\left(L_{n}^{2}\right)}\left\|a_{\Phi}\right\|_{L^{2}\left(P_{\Delta}, \mathbb{R}\right)}+K_{1} \sqrt{b-a}\left\|b_{\Phi}\right\|_{L^{2}\left(P_{\Delta}, \mathbb{R}\right)}\right)
\end{aligned}
$$

where

$$
K_{1}=\max \left\{\omega_{\Phi}(|u(t)|) ; t \in[a, b]\right\},
$$

and

$$
\begin{aligned}
& \left(\int_{a}^{b}|f(t, x(t), v(t))|^{2} d t\right)^{1 / 2} \\
& \leq\left(\int_{a}^{b}\left(a_{f}|x(t)|+b_{f}(t) \omega_{f}(|v(t)|)\right)^{2} d t\right)^{1 / 2} \\
& \leq \sqrt{2}\left(\int_{a}^{b}\left(a_{f}^{2}|x(t)|^{2}+\left(b_{f}(t)\right)^{2}\left(\omega_{f}(|v(t)|)\right)^{2}\right) d t\right)^{1 / 2} \\
& \leq \sqrt{2}\left(a_{f}^{2}\left(\gamma_{\alpha}\right)^{2}\|x\|_{I_{a+}^{\alpha}\left(L_{n}^{2}\right)}^{2}+K_{4}^{2}\left\|b_{f}\right\|_{L_{1}^{2}}^{2}\right)^{1 / 2} \\
& \leq \sqrt{2}\left(a_{f} \gamma_{\alpha}\|x\|_{I_{a+}^{\alpha}\left(L_{n}^{2}\right)}+K_{4}\left\|b_{f}\right\|_{L_{1}^{2}}\right)
\end{aligned}
$$

where

$$
K_{4}=\max \left\{\omega_{f}(|v(t)|) ; t \in[a, b]\right\}
$$


So,

$$
\begin{aligned}
|\varphi(x)|^{1 / 2} \geq & \frac{1}{\sqrt{2}}\left(\|x\|_{I_{a+}^{\alpha}\left(L_{n}^{2}\right)}-\sqrt{2} \gamma_{\alpha}\|x\|_{I_{a+}^{\alpha}\left(L_{n}^{2}\right)}\left\|a_{\Phi}\right\|_{L^{2}\left(P_{\Delta}, \mathbb{R}\right)}\right. \\
- & \sqrt{2} K_{1} \sqrt{b-a}\left\|b_{\Phi}\right\|_{L^{2}\left(P_{\Delta}, \mathbb{R}\right)} \\
& \left.-\sqrt{2} a_{f} \gamma_{\alpha}\|x\|_{I_{a+}^{\alpha}\left(L_{n}^{2}\right)}-\sqrt{2} K_{4}\left\|b_{f}\right\|_{L_{1}^{2}}\right) \\
= & \left(\frac{1}{\sqrt{2}}-\left\|a_{\Phi}\right\|_{L^{2}\left(P_{\Delta}, \mathbb{R}\right)} \gamma_{\alpha}-a_{f} \gamma_{\alpha}\right)\|x\|_{I_{a+}^{\alpha}\left(L_{n}^{2}\right)} \\
& -K_{1} \sqrt{b-a}\left\|b_{\Phi}\right\|_{L^{2}\left(P_{\Delta}, \mathbb{R}\right)}-K_{4}\left\|b_{f}\right\|_{L_{1}^{2}}
\end{aligned}
$$

for $x \in I_{a+}^{\alpha}\left(L_{n}^{2}\right)$. It means that $\varphi$ is coercive (cf., (4.1)).

In a standard way, we check that the differential $\varphi^{\prime}(x)$ of $\varphi$ at $x$ is given by

$$
\begin{aligned}
\varphi^{\prime}(x) h= & \int_{a}^{b}\left(D_{a+}^{\alpha} x(t)+\int_{a}^{t} \Phi(t, \tau, x(\tau), u(\tau)) d \tau-f(t, x(t), v(t))\right) \\
\times & \quad D_{a+}^{\alpha} h(t)+\int_{a}^{t} \Phi_{x}(t, \tau, x(\tau), u(\tau)) h(\tau) d \tau \\
& \left.-f_{x}(t, x(t), v(t)) h(t)\right) d t
\end{aligned}
$$

for $h \in I_{a+}^{\alpha}\left(L^{2}\right)$. Consequently, for any $x_{m}, x_{0} \in I_{a+}^{\alpha}\left(L_{n}^{2}\right)$, we have

$$
\begin{aligned}
\varphi^{\prime}\left(x_{m}\right)\left(x_{m}-x_{0}\right)= & \int_{a}^{b}\left(D_{a+}^{\alpha} x_{m}(t)+\int_{a}^{t} \Phi\left(t, \tau, x_{m}(\tau), u(\tau)\right) d \tau\right. \\
& \left.-f\left(t, x_{m}(t), v(t)\right)\right) \\
\times & \left(\left(D_{a+}^{\alpha} x_{m}(t)-D_{a+}^{\alpha} x_{0}(t)\right)\right. \\
& +\int_{a}^{t} \Phi_{x}\left(t, \tau, x_{m}(\tau), u(\tau)\right)\left(x_{m}(\tau)-x_{0}(\tau)\right) d \tau \\
\varphi^{\prime}\left(x_{0}\right)\left(x_{m}-x_{0}\right)= & \int_{a}^{b}\left(D_{a+}^{\alpha} x_{0}(t)\right. \\
& \left.+\int_{a}^{t} \Phi\left(t, \tau, x_{0}(\tau), u(\tau)\right) d \tau-f\left(t, x_{0}(t), v(t)\right)\right)
\end{aligned}
$$




$$
\begin{aligned}
& \times\left(\left(D_{a+}^{\alpha} x_{m}(t)-D_{a+}^{\alpha} x_{0}(t)\right)\right. \\
& \quad+\int_{a}^{t} \Phi_{x}\left(t, \tau, x_{0}(\tau), u(\tau)\right)\left(x_{m}(\tau)-x_{0}(\tau)\right) d \tau \\
& \left.\quad-f_{x}\left(t, x_{0}(t), v(t)\right)\left(x_{m}(t)-x_{0}(t)\right)\right) d t
\end{aligned}
$$

and

$$
\begin{aligned}
\varphi^{\prime}\left(x_{m}\right)\left(x_{m}-x_{0}\right)-\varphi^{\prime}\left(x_{0}\right)\left(x_{m}-x_{0}\right)= & \left\|x_{m}-x_{0}\right\|_{I_{a+}^{\alpha}\left(L_{n}^{2}\right)}^{2} \\
& +\sum_{i=1}^{14} \psi_{i}\left(x_{m}\right),
\end{aligned}
$$

where

$$
\begin{aligned}
& \psi_{1}\left(x_{m}\right)=\int_{a}^{b}\left(\int_{a}^{t} \Phi\left(t, \tau, x_{m}(\tau), u(\tau)\right) d \tau\right. \\
& \left.-\int_{a}^{t} \Phi\left(t, \tau, x_{0}(\tau), u(\tau)\right) d \tau\right)\left(D_{a+}^{\alpha} x_{m}(t)-D_{a+}^{\alpha} x_{0}(t)\right) d t \\
& \psi_{2}\left(x_{m}\right)=\int_{a}^{b}\left(f\left(t, x_{0}(t), v(t)\right)-f\left(t, x_{m}(t), v(t)\right)\right) \\
& \times\left(D_{a+}^{\alpha} x_{m}(t)-D_{a+}^{\alpha} x_{0}(t)\right) d t, \\
& \psi_{3}\left(x_{m}\right)=\int_{a}^{b} D_{a+}^{\alpha} x_{m}(t) \int_{a}^{t} \Phi_{x}\left(t, \tau, x_{m}(\tau), u(\tau)\right)\left(x_{m}(\tau)-x_{0}(\tau)\right) d \tau d t \\
& \psi_{4}\left(x_{m}\right)=-\int_{a}^{b} D_{a+}^{\alpha} x_{0}(t) \\
& \times \int_{a}^{t} \Phi_{x}\left(t, \tau, x_{0}(\tau), u(\tau)\right)\left(x_{m}(\tau)-x_{0}(\tau)\right) d \tau d t \\
& \psi_{5}\left(x_{m}\right)=\int_{a}^{b}\left(\int_{a}^{t} \Phi\left(t, \tau, x_{m}(\tau), u(\tau)\right) d \tau\right. \\
& \left.\times \int_{a}^{t} \Phi_{x}\left(t, \tau, x_{m}(\tau), u(\tau)\right)\left(x_{m}(\tau)-x_{0}(\tau)\right) d \tau\right) d t, \\
& \psi_{6}\left(x_{m}\right)=-\int_{a}^{b}\left(\int_{a}^{t} \Phi\left(t, \tau, x_{0}(\tau), u(\tau)\right) d \tau\right. \\
& \left.\times \int_{a}^{t} \Phi_{x}\left(t, \tau, x_{0}(\tau), u(\tau)\right)\left(x_{m}(\tau)-x_{0}(\tau)\right) d \tau\right) d t
\end{aligned}
$$




$$
\begin{aligned}
& \psi_{7}\left(x_{m}\right)=-\int_{a}^{b}\left(f\left(t, x_{m}(t), v(t)\right)\right. \\
& \left.\times \int_{a}^{t} \Phi_{x}\left(t, \tau, x_{m}(\tau), u(\tau)\right)\left(x_{m}(\tau)-x_{0}(\tau)\right) d \tau\right) d t \\
& \psi_{8}\left(x_{m}\right)=\int_{a}^{b}\left(f\left(t, x_{0}(t), v(t)\right)\right. \\
& \left.\times \int_{a}^{t} \Phi_{x}\left(t, \tau, x_{0}(\tau), u(\tau)\right)\left(x_{m}(\tau)-x_{0}(\tau)\right) d \tau\right) d t \\
& \psi_{9}\left(x_{m}\right)=-\int_{a}^{b} D_{a+}^{\alpha} x_{m}(t) f_{x}\left(t, x_{m}(t), v(t)\right)\left(x_{m}(t)-x_{0}(t)\right) d t, \\
& \psi_{10}\left(x_{m}\right)=\int_{a}^{b} D_{a+}^{\alpha} x_{0}(t) f_{x}\left(t, x_{0}(t), v(t)\right)\left(x_{m}(t)-x_{0}(t)\right) d t, \\
& \psi_{11}\left(x_{m}\right)=-\int_{a}^{b} \int_{a}^{t} \Phi\left(t, \tau, x_{m}(\tau), u(\tau)\right) d \tau \\
& \times f_{x}\left(t, x_{m}(t), v(t)\right)\left(x_{m}(t)-x_{0}(t)\right) d t, \\
& \psi_{12}\left(x_{m}\right)=\int_{a}^{b} \int_{a}^{t} \Phi\left(t, \tau, x_{0}(\tau), u(\tau)\right) d \tau \\
& \times f_{x}\left(t, x_{0}(t), v(t)\right)\left(x_{m}(t)-x_{0}(t)\right) d t, \\
& \psi_{13}\left(x_{m}\right)=\int_{a}^{b} f\left(t, x_{m}(t), v(t)\right) \\
& \times f_{x}\left(t, x_{m}(t), v(t)\right)\left(x_{m}(t)-x_{0}(t)\right) d t, \\
& \psi_{14}\left(x_{m}\right)=-\int_{a}^{b} f\left(t, x_{0}(t), v(t)\right) \\
& \times f_{x}\left(t, x_{0}(t), v(t)\right)\left(x_{m}(t)-x_{0}(t)\right) d t .
\end{aligned}
$$

We shall show that $\varphi$ satisfies the (PS) condition. Indeed, if $\left(x_{m}\right)$ is a (PS) sequence for $\varphi$, then the coercivity of $\varphi$ implies its boundedness. Consequently, there exists a subsequence $\left(x_{m_{k}}\right)$ which is weakly convergent in $I_{a+}^{\alpha}\left(L_{n}^{2}\right)$ to some $x_{0}$. Since (cf., [15, Lemma 3]) for any sequence $\left(x_{n}\right)$ weakly convergent in $I_{a+}^{\alpha}\left(L_{n}^{2}\right)$ to $x_{0},\left(x_{n}\right)$ is strongly convergent to $x_{0}$ in $L_{n}^{2}$ and the sequence of derivatives $\left(D_{a+}^{\alpha} x_{n}\right)$ is weakly convergent to $D_{a+}^{\alpha} x_{0}$ in $L_{n}^{2}$, therefore $x_{m_{k}} \rightarrow x_{0}$ in $L_{n}^{2}$ with respect to the norm and $D_{a+}^{\alpha} x_{m_{k}} \rightarrow D_{a+}^{\alpha} x_{0}$ weakly in $L_{n}^{2}$.

First, we shall show that $\psi_{i}\left(x_{m_{k}}\right) \underset{k \rightarrow \infty}{\rightarrow} 0$ for $i=1, \ldots, 14$. 
Let us consider the first term $\psi_{1}\left(x_{m_{k}}\right)$. In the same way as in the proof of Lemma 4.1, we check that

$$
\int_{a}^{\cdot}\left(\Phi\left(\cdot, \tau, x_{m_{k}}(\tau)\right)-\Phi\left(\cdot, \tau, x_{0}(\tau)\right)\right) d \tau \underset{m \rightarrow \infty}{\longrightarrow} 0
$$

in $L_{n}^{2}$. Consequently, $\psi_{1}\left(x_{m_{k}}\right)$ as a scalar product in $L_{n}^{2}$ of the functions $D_{a+}^{\alpha} x_{m}(\cdot)-D_{a+}^{\alpha} x_{0}(\cdot)$ and $\int_{a}^{\cdot}\left(\Phi\left(\cdot, \tau, x_{m_{k}}(\tau), u(\tau)\right)-\Phi\left(\cdot, \tau, x_{0}(\tau), u(\tau)\right)\right) d \tau$ tends to 0 as $k \rightarrow \infty$. Similarly, using the growth condition on $f$, we assert that $\psi_{2}\left(x_{m_{k}}\right) \rightarrow 0$. Convergence of $\psi_{i}\left(x_{m_{k}}\right)$ to 0 for $i=3, \ldots, 14$ follows from the convergence of $x_{m_{k}}(\cdot)$ to $x_{0}(\cdot)$ in $L^{2}$.

Since $\varphi^{\prime}\left(x_{0}\right)$ is linear and continuous functional on $I_{a+}^{\alpha}\left(L_{n}^{2}\right)$, convergence of $\varphi^{\prime}\left(x_{0}\right)\left(x_{m_{k}}-x_{0}\right)$ to 0 follows directly from the weak convergence $x_{m_{k}} \rightarrow x_{0}$ in $I_{a+}^{\alpha}\left(L_{n}^{2}\right)$.

Convergence of $\varphi^{\prime}\left(x_{m_{k}}\right)\left(x_{m_{k}}-x_{0}\right)$ to 0 follows from the estimation

$$
\left|\varphi^{\prime}\left(x_{m_{k}}\right)\left(x_{m_{k}}-x_{0}\right)\right| \leq\left\|\varphi^{\prime}\left(x_{m_{k}}\right)\right\|_{\mathcal{L}\left(I_{a+}^{\alpha}\left(L_{n}^{2}\right), \mathbb{R}\right)}\left\|x_{m_{k}}-x_{0}\right\|_{I_{a+}^{\alpha}\left(L_{n}^{2}\right)},
$$

boundedness of the sequence $\left(x_{m_{k}}\right)$ in $I_{a+}^{\alpha}\left(L_{n}^{2}\right)$ and convergence of $\varphi^{\prime}\left(x_{m_{k}}\right)$ to 0.

Thus, $x_{m_{k}} \rightarrow x_{0}$ in $I_{a+}^{\alpha}\left(L_{n}^{2}\right)$ with respect to the norm.

So, all assumptions of the global implicit function theorem are satisfied. Consequently, for any $(u, v) \in L_{m}^{\infty} \times L_{r}^{\infty}$, there exists a unique solution $x_{u, v} \in I_{a+}^{\alpha}\left(L_{n}^{2}\right)$ of the problem (1.2), the mapping

$$
\lambda: L_{m}^{\infty} \times L_{r}^{\infty} \ni(u, v) \longmapsto x_{u, v} \in I_{a+}^{\alpha}\left(L_{n}^{2}\right)
$$

is continuous differentiable in the Frechet sense on $L_{m}^{\infty} \times L_{r}^{\infty}$ and the differential $\lambda^{\prime}(u, v)$ at a point $(u, v) \in L_{m}^{\infty} \times L_{r}^{\infty}$ is the following

$$
\lambda^{\prime}(u, v): L_{m}^{\infty} \times L_{r}^{\infty} \ni(f, g) \longmapsto z_{f, g} \in I_{a+}^{\alpha}\left(L_{n}^{2}\right),
$$

where $z_{f, g}$ is such that

$$
\begin{aligned}
& D_{a+}^{\alpha} z_{f, g}(t)+\int_{a}^{t} \Phi_{x}\left(t, \tau, x_{u, v}(\tau), u(\tau)\right) z_{f, g}(\tau) d \tau \\
& \quad-f_{x}\left(t, x_{u, v}(t), v(t)\right) z_{f, g}(t) \\
& =-\int_{a}^{t} \Phi_{u}\left(t, \tau, x_{u, v}(\tau), u(\tau)\right) f(\tau) d \tau+f_{v}\left(t, x_{u, v}(t), v(t)\right) g(t)
\end{aligned}
$$

almost everywhere on $J$. 
Remark 4.3. Let us point out that, if we replace the growth condition on $\Phi_{x}$ from $(A 2)$ with the following one

$$
\left|\Phi_{x}(t, \tau, x, u)\right| \leq c_{\Phi}(t, \tau) \omega_{\Phi}(|u|)
$$

for $(t, \tau) \in P_{\Delta}$ almost everywhere, $x \in \mathbb{R}^{n}, u \in \mathbb{R}^{m}$, where $c_{\Phi} \in$ $L^{2}\left(P_{\Delta}, \mathbb{R}_{0}^{+}\right), \omega_{\Phi} \in C\left(\mathbb{R}_{0}^{+}, \mathbb{R}_{0}^{+}\right)$, assuming additionally that

$$
\int_{a}^{t} c_{\Phi}^{2}(t, \tau) d \tau \leq C
$$

for $t \in J$ almost everywhere and some $C>0$, then the existence of a unique solution $x_{u, v}$ of (1.2), for any fixed $(u, v)$, can be deduced from the results contained in the Appendix (with the aid of the mean value theorem and without a condition of type (4.1)). Applying, additionally, the local implicit function theorem, one can obtain continuous differentiability in Frechet sense of the mapping $L_{m}^{\infty} \times L_{r}^{\infty} \ni(u, v) \mapsto x_{u, v} \in$ $I_{a+}^{\alpha}\left(L_{n}^{2}\right)$.

5. Example. Let us fix $\alpha \in(0,1), C>0, E>0$, and consider the following problem

$$
\left\{\begin{array}{l}
D_{a+}^{\alpha} x(t)+\int_{a}^{t} C \sqrt{t-\tau} \ln \left(\sqrt{(x(\tau))^{2}+1}+(u(\tau))^{2}\right) d \tau \\
=E \sqrt[3]{t} \sin (x(t)+1+v(t)), t \in[0,1] \text { almost everywhere } \\
I_{a+}^{1-\alpha} x(a)=0
\end{array}\right.
$$

where $x \in I_{a+}^{\alpha}\left(L^{2}([0,1], \mathbb{R})\right), u \in L^{\infty}([0,1], \mathbb{R})$ and $v \in L^{\infty}([0,1], \mathbb{R})$. So, it is a particular case of problem (1.2) with the functions

$$
\begin{gathered}
\Phi: P_{\Delta} \times \mathbb{R} \times \mathbb{R} \rightarrow \mathbb{R}, \\
\Phi(t, \tau, x, u)=C \sqrt{t-\tau} \ln \left(\sqrt{x^{2}+1}+u^{2}\right)
\end{gathered}
$$

where $\left.P_{\Delta}=\{(t, \tau) \in[0,1] \times 0,1] ; \tau \leq t\right\}$ and

$$
\begin{gathered}
f:[0,1] \times \mathbb{R} \times \mathbb{R} \rightarrow \mathbb{R}, \\
f(t, x, v)=E \sqrt[3]{t} \sin (x+1+v) .
\end{gathered}
$$

Of course, these functions are measurable in $(t, \tau) \in P_{\Delta}, t \in[0,1]$, respectively, and continuous differentiable with respect to $(x, u) \in$ $\mathbb{R} \times \mathbb{R},(x, v) \in \mathbb{R} \times \mathbb{R}$, respectively. Moreover, using the inequalities 
$\ln s \leq \sqrt{s}$ and $\sqrt{a+b} \leq \sqrt{a}+\sqrt{b}$, we obtain

$$
\begin{aligned}
|\Phi(t, \tau, x, u)| & =\left|C \sqrt{t-\tau} \ln \left(\sqrt{x^{2}+1}+u^{2}\right)\right| \\
& \leq C \sqrt{t-\tau} \ln \left(\sqrt{x^{2}}+1+u^{2}\right) \\
& \leq C \sqrt{t-\tau} \sqrt{\sqrt{x^{2}}+1+u^{2}} \\
& \leq C \sqrt{t-\tau}\left(\sqrt{\sqrt{x^{2}}}+\sqrt{1+u^{2}}\right) \\
& \leq C \sqrt{t-\tau}\left(\sqrt{|x|}+\sqrt{1+u^{2}}\right) \\
& \leq C \sqrt{t-\tau}\left(|x|+1+\sqrt{1+u^{2}}\right) \\
& =C \sqrt{t-\tau}|x|+C \sqrt{t-\tau}\left(\sqrt{1+|u|^{2}}+1\right) \\
& \leq C \sqrt{t-\tau}|x|+C \sqrt{t-\tau} \max \left\{\left(\sqrt{1+|u|^{2}}+1\right), 2|u|\right\}
\end{aligned}
$$

and

$$
\begin{aligned}
\left|\Phi_{x}(t, \tau, x, u)\right|= & C \sqrt{t-\tau} \frac{|x|}{\left(\sqrt{x^{2}+1}+u^{2}\right) \sqrt{x^{2}+1}} \\
\leq & C|x| \frac{1}{\left(\sqrt{x^{2}+1}+u^{2}\right) \sqrt{x^{2}+1}} \\
\leq & C|x| \leq C|x| \max \left\{\left(\sqrt{1+|u|^{2}}+1\right), 2|u|\right\}\left|\Phi_{u}(t, \tau, x, u)\right| \\
= & C \sqrt{t-\tau} \frac{2|u|}{\sqrt{x^{2}+1}+u^{2}} \leq C \sqrt{t-\tau} 2|u| \\
\leq & C \sqrt{t-\tau} \max \left\{\left(\sqrt{1+|u|^{2}}+1\right), 2|u|\right\} \\
\leq & C \sqrt{t-\tau}|x| \\
& +C \sqrt{t-\tau} \max \left\{\left(\sqrt{1+|u|^{2}}+1\right), 2|u|\right\}
\end{aligned}
$$

for $(t, \tau) \in P_{\Delta},(x, u) \in \mathbb{R} \times \mathbb{R}$. So, the growth conditions concerning $\Phi$ are satisfied with

$$
\begin{gathered}
a_{\Phi}(t, \tau)=b_{\Phi}(t, \tau)=C \sqrt{t-\tau}, \quad C_{\Phi}=C, \\
\omega_{\Phi}(r)=\max \left\{\sqrt{1+r^{2}}+1,2 r\right\} .
\end{gathered}
$$


Let us observe that

$$
\left\|a_{\Phi}\right\|_{L^{2}\left(P_{\Delta}, \mathbb{R}\right)}=\frac{C}{\sqrt{6}}
$$

Similarly,

$$
\begin{aligned}
|f(t, x, v)| & =E \sqrt[3]{t}|\sin (x+1+v)| \\
& \leq E \sqrt[3]{t} \mid x+1+v) \mid \\
& \leq E \sqrt[3]{t}|x|+E \sqrt[3]{t}(|v|+1) \\
& \leq E|x|+E \sqrt[3]{t}(|v|+1)
\end{aligned}
$$

and

$$
\begin{aligned}
\left|f_{x}(t, x, v)\right| & =E \sqrt[3]{t}|\cos (x+1+v)| \\
& \leq E \leq E(|v|+1), \\
\left|f_{v}(t, x, v)\right| & =E \sqrt[3]{t}|\cos (x+1+v)| \\
& \leq E \sqrt[3]{t} \leq E \sqrt[3]{t}(|v|+1) \\
& \leq E|x|+E \sqrt[3]{t}(|v|+1)
\end{aligned}
$$

for $t \in[0,1],(x, u) \in \mathbb{R} \times \mathbb{R}$. Thus, the growth conditions concerning $f$ are satisfied with

$$
\begin{gathered}
a_{f}=d_{f}=E, \quad b_{f}(t)=E \sqrt[3]{t}, \\
\omega_{f}(r)=r+1 .
\end{gathered}
$$

Choosing the constants $C$ and $E$ such that

$$
\frac{1}{\Gamma(\alpha+1)}\left(\frac{C}{\sqrt{6}}+E\right)<\frac{1}{\sqrt{2}}
$$

we see that (4.1) is satisfied (using MAPLE one can check that, for example, constants $\alpha=1 / 2$ and $C=E=1 / 3$ satisfy the above inequality).

Consequently, for any pair of functional parameters $u \in L^{\infty}([0,1], \mathbb{R})$, $v \in L^{\infty}([0,1], \mathbb{R})$, there exists a unique solution $x_{u, v}$ to problem $(5.1)$, in the space $I_{a+}^{\alpha}\left(L_{n}^{2}\right)$, and the mapping

$$
L^{\infty}([0,1], \mathbb{R}) \times L^{\infty}([0,1], \mathbb{R}) \ni(u, v) \longrightarrow x_{u, v} \in I_{a+}^{\alpha}\left(L_{n}^{2}\right)
$$

is continuous differentiable. 
6. Appendix. Let us consider the following system:

$$
D_{a+}^{\alpha} x(t)+\int_{a}^{t} \Psi(t, \tau, x(\tau)) d \tau=g(t, x(t)), \quad t \in J \text { a.e., }
$$

where $\Psi: P_{\Delta} \times \mathbb{R}^{n} \rightarrow \mathbb{R}^{n}, g: J \times \mathbb{R}^{n} \rightarrow \mathbb{R}^{n}$ and $x \in I_{a+}^{\alpha}\left(L_{n}^{2}\right)$. On the functions $\Psi$ and $g$ we assume that

- $\Psi(\cdot, \cdot, x)$ is measurable on $P_{\Delta}$ for any $x \in \mathbb{R}^{n}$, and

$$
\left|\Psi\left(t, \tau, x_{1}\right)-\Psi\left(t, \tau, x_{2}\right)\right| \leq d(t, \tau)\left|x_{1}-x_{2}\right|
$$

for $(t, \tau) \in P_{\Delta}$ almost everywhere, $x_{1}, x_{2} \in \mathbb{R}^{n}$, where $d \in$ $L^{2}\left(P_{\Delta}, \mathbb{R}^{n}\right)$ and

$$
\int_{a}^{t} d^{2}(t, s) d s \leq D
$$

for $t \in J$ almost everywhere and some $D>0$;

- $\Psi(\cdot, \cdot, 0) \in L^{2}\left(P_{\Delta}, \mathbb{R}^{n}\right)$;

- $g(\cdot, x)$ is measurable on $J$ for any $x \in \mathbb{R}^{n}$, and

$$
\left|g\left(t, x_{1}\right)-g\left(t, x_{2}\right)\right| \leq L\left|x_{1}-x_{2}\right|
$$

for $t \in J$ almost everywhere, $x_{1}, x_{2} \in \mathbb{R}^{n}$, where $L>0$ is some constant;

- $g(\cdot, 0) \in L_{n}^{2}$.

It is easy to see that the existence of a unique solution $x$ to system (6.1) in the space $I_{a+}^{\alpha}\left(L_{n}^{2}\right)$ is equivalent to the existence of a unique solution $l$ to system

$$
l(t)+\int_{a}^{t} \Psi\left(t, \tau, I_{a+}^{\alpha} l(\tau)\right) d \tau=g\left(t, I_{a+}^{\alpha} l(t)\right), \quad t \in J \text { a.e. }
$$

in the space $L_{n}^{2}$; in such a case, $D_{a+}^{\alpha} x=l$. We have:

Lemma 6.1. There exists a unique fixed point of the operator

$$
T: L_{n}^{2} \ni l \longmapsto g\left(t, I_{a+}^{\alpha} l(t)\right)-\int_{a}^{t} \Psi\left(t, \tau, I_{a+}^{\alpha} l(\tau)\right) d \tau \in L_{n}^{2} .
$$

Proof. Operator $T$ is well-posed. Indeed, the squared integrability of the first term follows from the Lipschitz condition posed on $g$. The fact that the second term belongs to $L_{n}^{2}$ can be checked as in the proof 
of Lemma 4.1. We shall show that there exists a positive integer $k$ such that the operators

$$
\begin{aligned}
& T_{g}: L_{n}^{2} \ni l \longmapsto g\left(t, I_{a+}^{\alpha} l(t)\right) \in L_{n}^{2} \\
& T_{\Psi}: L_{n}^{2} \ni l \longmapsto \int_{a}^{t} \Psi\left(t, \tau, I_{a+}^{\alpha} l(\tau)\right) d \tau \in L_{n}^{2}
\end{aligned}
$$

are contracting with constants $\xi_{1}, \xi_{2} \in(0,1 / 2)$, respectively, provided that $L_{n}^{2}$ is considered with the Bielecki norm

$$
\|l\|_{k}=\left(\int_{a}^{1} e^{-k t}|l(t)|^{2} d t\right)^{1 / 2}, \quad l \in L_{n}^{2} .
$$

Indeed, let us fix $k \in \mathbb{N}$. In [17], it is shown that

$$
\begin{aligned}
\left\|T_{g}\left(l_{1}\right)-T_{g}\left(l_{2}\right)\right\|_{k}^{2} & =\int_{a}^{b} e^{-k t}\left|g\left(t, I_{a+}^{\alpha} l_{1}(t)\right)-g\left(t, I_{a+}^{\alpha} l_{2}(t)\right)\right|^{2} d t \\
& \leq \gamma_{\alpha}\left(L^{2} /(2 k)^{\alpha}\right)\left\|l_{1}-l_{2}\right\|_{k}^{2}
\end{aligned}
$$

for $l_{1}, l_{2} \in L_{n}^{2}$. In [15, Proof of Lemma 7], it is shown that

$$
\left\|T_{\Psi}\left(l_{1}\right)-T_{\Psi}\left(l_{2}\right)\right\|_{k}^{2} \leq\left(\gamma_{\alpha}\right)^{2}(D / k)\left\|l_{1}-l_{2}\right\|_{k}^{2}
$$

for $l_{1}, l_{2} \in L_{n}^{2}$. So, it is sufficient to choose $k$ such that

$$
\max \left\{L \sqrt{\gamma_{\alpha} /(2 k)^{\alpha}}, \gamma_{\alpha} \sqrt{(D / k)}\right\}<\frac{1}{2},
$$

and the proof is completed.

Acknowledgments. The authors wish to express their thanks to the anonymous referee for his valuable remarks.

\section{REFERENCES}

1. A. Aghajani, Y. Jalilian and J.J. Trujillo, On the existence of solutions of fractional integro-differential equations, Fract. Calc. Appl. Anal. 15 (2012), 44-69.

2. B. Ahmad and J. Nieto, Riemann-Liouville fractional integro-differential equations with fractional nonlocal integral boundary conditions, Bound. Value Prob. 2011 (2011), 36.

3. L. Bourdin, Existence of a weak solution for fractional Euler-Lagrange equations, J. Math. Anal. Appl. 399 (2013), 239-251. 
4. L. Bourdin and D. Idczak, Fractional fundamental lemma and fractional integration by parts formula-Applications to critical points of Bolza functionals and to linear boundary value problems, Adv. Differ. Equat. 20 (2015), 213-232.

5. H. Brezis, Analyse Fonctionelle. Theorie et Applications, Masson, Paris, 1983.

6. S. Bushnaq, S. Momani and Y. Zhou, A reproducing kernel Hilbert space method for solving integro-differential equations of fractional order, J. Optim. Theor. Appl. (2012), doi: 10.1007/s10957-012-0207-2.

7. M. Galewski and M. Koniorczyk, On a global diffeomorphism between two Banach spaces and some application, Stud. Sci. Math. Hung. 52 (2015), 65-86.

8. B. Gayathri, R. Murugesu and J. Rajasingh, Existence of solutions of some impulsive fractional integrodifferential equations, Int. J. Math. Anal. 6 (2012), 825836.

9. G.H. Hardy and J.E. Littlewood, Some properties of fractional integrals, Proc. Lond. Math. Soc. 24 (1925), 37-41.

10. D. Idczak, A global implicit function theorem and its applications to functional equations, Discr. Cont. Dynam. Syst. 19 (2014), 2549-2556.

11. , On a generalization of a global implicit function theorem, Advanced Nonlinear Studies, accepted, doi:10.1515/ans-2015-5008.

12. D. Idczak and M. Majewski, Fractional fundamental lemma of order $\alpha \in$ $\left(n-\frac{1}{2}, n\right)$ with $n \in N, n \geq 2$, Dyn. Syst. Appl. 21 (2012), 251-268.

13. D. Idczak and A. Rogowski, On a generalization of Krasnoselskii's theorem, J. Austr. Math. Soc. 72 (2002), 389-394.

14. D. Idczak, A. Skowron and S. Walczak, On the diffeomorphisms between Banach and Hilbert spaces, Adv. Nonlin. Stud. 12 (2012), 89-100.

15. Sensitivity of a fractional integrodifferential Cauchy problem of Volterra type, Abstr. Appl. Anal. 2013 (2013), Article ID 129478.

16. D. Idczak and S. Walczak, Compactness of fractional imbeddings, Proc. 17th Inter. Conf. Methods and Models in Automation and Robotics, Miedzyzdroje, Poland, 2012, 585-588.

17. R. Kamocki, Pontryagin maximum principle for fractional ordinary optimal control problems, Math. Meth. Appl. Sci. 37 (2014), DOI: 10.1002/mma.2928.

18. A.A. Kilbas, H.M. Srivastava and J.J. Trujillo, Theory and applications of fractional differential equations, Elsevier, Amsterdam, 2006.

19. M.M. Matar, Boundary value problem for some fractional integrodifferential equations with nonlocal conditions, Inter. J. Nonlin. Sci. 11 (2011), 3-9.

20. D. Nazari and S. Shahmorad, Application of the fractional differential transform method to fractional-order integro-differential equations with nonlocal boundary conditions, J. Comp. Appl. Math. 234 (2010), 883-891.

21. S.G. Samko, A.A. Kilbas and O.I. Marichev, Fractional integrals and derivatives-Theory and applications, Gordon and Breach, Amsterdam, 1993.

22. W. Sudsutad and J. Tariboon, Existence results of fractional integrodifferential equations with m-point multi-term fractional order integral boundary conditions, Bound. Value Prob. (2012), 94. 
23. J. Wang and $\mathrm{W}$. Wei, Nonlinear delay integrodifferential systems with Caputo fractional derivative in infinite-dimensional spaces, Ann. Polon. Math. 105 (2012), 209-223.

24. R. Wheeden and A. Zygmund, Measure and integral. An introduction to real analysis, Marcel Dekker, New York, 1977.

25. W. Wittayakiattilerd and A. Chonwerayuth, Fractional integro-differential equations of mixed type with solution operator and optimal controls, J. Math. Res. 3 (2011), 140-151.

26. Z. Yan, Existence results for fractional functional integrodifferential equations with nonlocal conditions in Banach spaces, Ann. Polon. Math. 97 (2010), $285-299$.

Faculty of Mathematics and Computer Science, University of Lodz, BaNACHA 22, 90-238 Lodz, POLAND

Email address: idczak@math.uni.lodz.pl

Faculty of Mathematics and Computer Science, University of Lodz, BaNACHA 22, 90-238 Lodz, POLAND

Email address: stawal@math.uni.lodz.pl 